\title{
A MACROSCOPIC TRAFFIC FLOW MODEL WITH FINITE BUFFERS ON NETWORKS: WELL-POSEDNESS BY MEANS OF HAMILTON-JACOBI EQUATIONS
}

NICOLAS LAURENT-BROUTY*, ALEXANDER KEIMER ${ }^{\dagger}$, PAOLA GOATIN $^{\ddagger}$, AND

ALEXANDRE M. BAYEN§

\begin{abstract}
We introduce a model dealing with conservation laws on networks and coupled boundary conditions at the junctions. In particular, we introduce buffers of fixed arbitrary size and timedependent split ratios at the junctions, which represent how traffic is routed through the network, while guaranteeing spill-back phenomena at nodes. Having defined the dynamics at the level of conservation laws, we lift it up to the Hamilton-Jacobi (H-J) formulation and write boundary datum of incoming and outgoing junctions as functions of the queue sizes and vice-versa. The Hamilton-Jacobi formulation provides the necessary regularity estimates to derive a fixed-point problem in a proper Banach space setting, which is used to prove well-posedness of the model. Finally, we detail how to apply our framework to a non-trivial road network, with several intersections and finite-length links.
\end{abstract}

Keywords. Macroscopic traffic flow models; networks; buffers; fixed-point; Hamilton-Jacobi equations; conservation laws; LWR model.

AMS subject classifications. 35L65; 35R02; 35F21; 35L04;

\section{Introduction}

The first application of conservation laws to traffic flow modeling dates back to the mid 1950s, with the seminal Lighthill-Whitham-Richards (LWR) model [35,37]. The model consists of a single conservation law accounting for the conservation of vehicles:

$$
\partial_{t} \rho+\partial_{x}(\rho v(\rho))=0, \quad x \in \mathbb{R}, t>0,
$$

where $\rho=\rho(t, x)$ denotes the density of vehicles on the road and $v=v(\rho)$ represents the mean velocity of the flow. The properties of this fairly simple model are now wellknown, and it is still widely used to model traffic. The main difficulty lies in modeling the behavior of traffic at junctions, which is not mathematically clear, due to phenomena like traveling congestion on incoming and outgoing roads and allocation of traffic on outgoing roads. Modeling traffic on road networks is essential in order to address the Dynamic Traffic Assignment (DTA) problem, which consists in optimizing trajectories of vehicles on networks in order to reduce travel times and congestion. Mathematically, the difficulty lies in defining the appropriate boundary conditions at intersections, in order to provide existence and uniqueness of solutions. To treat this difficulty, different solutions have been proposed in the literature. Two main approaches can be identified. The first consists in defining Riemann solvers at the junctions [22, Section 3], which are mappings that provide solutions to Cauchy problems with constant initial data on each link. Once these mappings are defined, wave-front-tracking or finite volume schemes enable one to build solutions to more general Cauchy problems. The main limitation of this approach is that the solution does not necessarily depend in a Lipschitz continuous way on the initial datum, as pointed out in [24, Section 5] and [12]. The other approach

\footnotetext{
*Université Côte d'Azur, Inria, CNRS, LJAD, France and Ecole des Ponts ParisTech, Champs-surMarne, France, nicolas.laurent-brouty@inria.fr

${ }^{\dagger}$ Institute of Transportation Studies, University of California, Berkeley, CA, keimer@berkeley.edu

${ }^{\ddagger}$ Inria Sophia Antipolis - Méditerranée, Université Côte d'Azur, Inria, CNRS, LJAD, paola.goatin@inria.fr

$\S$ Institute of Transportation Studies, University of California, Berkeley, CA, bayen@berkeley.edu
} 
is to couple incoming and outgoing links by a buffer located at the junction. The state of this buffer is then governed by an ordinary differential equation, taking into account the boundary conditions at the junction so that conservation of mass is guaranteed. This modeling framework was first introduced for supply-chain networks in $[5,26,27,29]$ and then adapted to traffic flow on networks in [9-11,21,25,30]. This approach provides stability estimates which are crucial from a control point of view, but may lead to a potential loss of information at the junctions, depending on how the buffer is modeled and whether one aggregates commodities at the junction level or not. The mentioned drawbacks of existing models (missing regularity or loss of information) prevent from implementing control strategies at the intersection level, mandatory to address the DTA problem.

We develop a control framework by implementing time-varying routing functions at junctions, which assign a ratio of the incoming flow to the outgoing edges. Depending on the capacity of outgoing links and the values of these ratios, incoming flows might not be fully assignable to outgoing roads at specific times. We then implement a buffer at the entrance of any outgoing road, with arbitrary chosen limited capacity, which takes into account the possible exceed on demand. Once the buffer has reached its limited capacity, the unsatisfied demand impacts the incoming roads, so that back-travelling phenomena are intrinsically treated.

To address well-posedness of the model, we transform the described problem into a fixed-point problem at the level of Hamilton-Jacobi partial differential equations (H-J PDEs), relying on the higher regularity of solutions. For the general theory of HamiltonJacobi equations we refer the reader to $[3,17,32]$ and to $[6,14,15,20]$ for applications to traffic flow modeling. Hamilton-Jacobi equations on networks without buffers were developed in [1,31]. Our analysis strongly relies on previous contributions in [9-11], where a similar fixed-point problem was posed. In these articles, the authors assume that the routing of each population is predetermined initially, and use transport equations to propagate this information and to keep track of different populations having different routes. On the other hand, we propose an approach which enables real-time routing modifications, by enforcing time-varying routing functions at junctions. For other approaches to traffic routing in a macroscopic non-stationary setting, we refer the reader to $[18,23,38]$.

This paper provides a rigorous well-posedness result for every finite buffer size, when changing involved input data in the proper topology. This enables us to study optimal control problems in which we control the routing parameters at junctions in an optimal way assuming a uniform $B V$ bound on the routing, a reasonable assumption as the change of traffic flow should not be too irregular. We also detail how the prototype junction model, defined on semi-infinite incoming and outgoing links, can be generalized to realistic networks. The main idea here is to use the finite propagation speed of information, so that one can decouple the fixed-point problems at the different junctions for sufficiently small times.

1.1. Outline. In Section 2, we introduce the model, explain the dynamics governing links and buffers and explain how to derive the Hamilton-Jacobi formulation of the problem, starting from the conservation law formulation. In Section 3, we propose a rigorous definition of solutions, based on the literature and on the modeling assumptions. After recalling some fundamental results about Hamilton-Jacobi equations in Section 4.1, we define the Banach fixed-point problem in Section 4.2 and study its properties in Section 4.3. In Section 4.4, we show the existence and uniqueness of a fixed-point, and thus the well-posedness of the Hamilton-Jacobi formulation of the 
problem. Section 5 presents results concerning the stability of solutions when changing the routing, initial datum of incoming and outgoing roads and the initial state of the buffers. In Section 6 we show that our framework enables us to write an optimal control problem w.r.t. routing, and to prove the existence of a minimizer. In Section 7 we detail how to use the framework on a physical road network, containing several intersections and finite-length links, providing explicit solutions to the Hamilton-Jacobi formulation. Finally, Section 8 presents the conclusions and suggests additional and future topics for research.

\section{The Model}

In this section, we present the dynamical model on the network. We first introduce an archetype network and then define the link dynamics (governed by the LWR PDE) and the node dynamics which are determined by the boundary data of the incoming and outgoing roads as well as the state of the buffer at the considered intersection. Note that the topology of the considered archetype network is sufficient to generalize to arbitrary connected and directed graphs.

2.1. Network. We consider as archetype network a single node $v$ with incoming $\mathcal{I}$ and outgoing $\mathcal{O}$ links as illustrated in Fig. 2.1. For simplicity, we first assume that each entering link $i \in \mathcal{I}$ is characterized by the spatial segment $(-\infty, 0]$. Similarly, each exiting link $j \in \mathcal{O}$ is characterized by the open segment $[0, \infty)$ so that we assume semiinfinite roads. In Section 7 we will discuss how we can generalize the proposed dynamics to general networks in a straightforward manner.

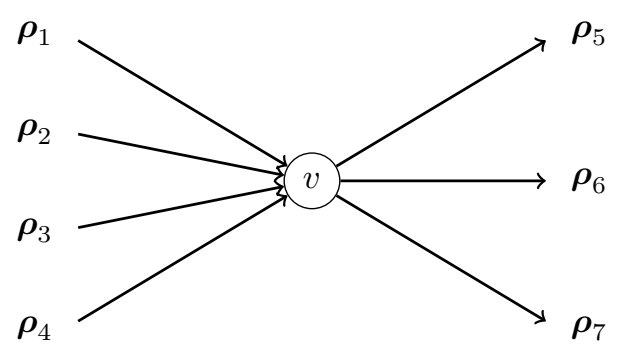

FIG. 2.1. Illustration of the archetype network, a node with incoming $\mathcal{I}:=\{1,2,3,4\}$ and outgoing $\mathcal{O}:=\{5,6,7\}$ links.

2.2. Link Dynamics. We denote by $\boldsymbol{\rho}_{i}(t, x), i \in \mathcal{I}$, the density of vehicles on the incoming links at space-time coordinate $(t, x) \in(0, T) \times \mathbb{R}_{<0}$ and by $\boldsymbol{\rho}_{j}(t, x)$ the density on the outgoing links $j \in \mathcal{O}$ at space-time coordinate $(t, x) \in(0, T) \times \mathbb{R}_{>0}$ for a given and fixed time horizon $T \in \mathbb{R}_{>0}$.

On each link of the network, we assume that the density of vehicles satisfies the LWR model for given specific flux functions so that the dynamics read for $i \in \mathcal{I}$ and $j \in \mathcal{O}$

$$
\begin{aligned}
\partial_{t} \boldsymbol{\rho}_{i}(t, x)+\partial_{x} \mathbf{f}_{i}\left(\boldsymbol{\rho}_{i}(t, x)\right) & =0, & & (t, x) \in(0, T) \times \mathbb{R}_{<0}, \\
\boldsymbol{\rho}_{i}(0, x) & =\boldsymbol{\rho}_{0, i}^{\text {in }}(x), & & x \in \mathbb{R}_{<0}, \\
\partial_{t} \boldsymbol{\rho}_{j}(t, x)+\partial_{x} \mathbf{f}_{j}\left(\boldsymbol{\rho}_{j}(t, x)\right) & =0, & & (t, x) \in(0, T) \times \mathbb{R}_{>0}, \\
\boldsymbol{\rho}_{j}(0, x) & =\boldsymbol{\rho}_{0, j}^{\text {out }}(x), & & x \in \mathbb{R}_{>0} .
\end{aligned}
$$


Assumption 2.1 (Assumptions on the flux function). We assume that the initial densities are bounded, i.e.

$$
\boldsymbol{\rho}_{0, i}^{\text {in }} \in\left[0, \boldsymbol{\rho}_{i}^{\max }\right], \quad \boldsymbol{\rho}_{0, j}^{\text {out }} \in\left[0, \boldsymbol{\rho}_{j}^{\max }\right]
$$

for some $\boldsymbol{\rho}_{i}^{\max } \in \mathbb{R}_{>0}, i \in \mathcal{I}, \boldsymbol{\rho}_{j}^{\max } \in \mathbb{R}_{>0}, j \in \mathcal{O}$. Moreover, we assume that the flux functions $\mathbf{f}_{i}, \mathbf{f}_{j}$ are smooth and strictly concave:

$$
\begin{array}{ccc}
\forall i \in \mathcal{I}: \mathbf{f}_{i} \in C^{2}\left(\left[0, \boldsymbol{\rho}_{i}^{\max }\right]\right), & \mathbf{f}_{i}^{\prime \prime}(z)<0 \forall z \in\left[0, \boldsymbol{\rho}_{i}^{\max }\right], & \mathbf{f}_{i}(0)=\mathbf{f}_{i}\left(\boldsymbol{\rho}_{i}^{\max }\right)=0, \\
\forall j \in \mathcal{O}: \mathbf{f}_{j} \in C^{2}\left(\left[0, \boldsymbol{\rho}_{j}^{\max }\right]\right), & \mathbf{f}_{j}^{\prime \prime}(z)<0 \forall z \in\left[0, \boldsymbol{\rho}_{j}^{\max }\right], & \mathbf{f}_{j}(0)=\mathbf{f}_{j}\left(\boldsymbol{\rho}_{j}^{\max }\right)=0 .
\end{array}
$$

We define the critical densities $\boldsymbol{\rho}_{i}^{\text {crit }}, \boldsymbol{\rho}_{j}^{\text {crit }}$ such that:

$$
\boldsymbol{\rho}_{i}^{\text {crit }} \in\left[0, \boldsymbol{\rho}_{i}^{\max }\right]: \mathbf{f}_{i}^{\prime}\left(\boldsymbol{\rho}_{i}^{\text {crit }}\right)=0, \quad \boldsymbol{\rho}_{j}^{\text {crit }} \in\left[0, \boldsymbol{\rho}_{j}^{\max }\right]: \mathbf{f}_{j}^{\prime}\left(\boldsymbol{\rho}_{j}^{\text {crit }}\right)=0
$$

2.3. Node Dynamics. The node dynamics are realized by a buffer and the boundary flux of incoming and outgoing roads. We present three different models for the buffer:

- the Single-Buffer/Multi-Queues in Section 2.3.1,

- the Single-Buffer/Single-Queue in Section 2.3.2,

- the Independent-Buffers in Section 2.3.3.

2.3.1. Single-Buffer/Multi-Queues. In the Single-Buffer/Multi-Queues scenario, for each $j \in \mathcal{O}$ and any $t \in[0, T]$ we define a queue state $\mathbf{q}_{j}(t) \geq 0$ which describes the number of vehicles queued before entering link $j$ serving all incoming links $i \in \mathcal{I}$. In addition, we assume that the intersection can store a maximum quantity of vehicles $M \in \mathbb{R}_{>0}$, which corresponds to a buffer size. The space left in the buffer at any time $t$ is then $M-\sum_{j \in \mathcal{O}} \mathbf{q}_{j}(t)$.

For each incoming link $i \in \mathcal{I}$, we define a time-dependent priority coefficient $\mathbf{c}_{i} \in$ $L^{\infty}\left((0, T) ; \mathbb{R}_{\geq 0}\right)$ which denotes the order of priority given to the entry in the junction. We then set the boundary incoming flux on any link $i \in \mathcal{I}$ such that it minimizes the demand from traffic and the supply of space in the buffer for $t \in(0, T)$ :

$$
\boldsymbol{\Gamma}_{i}(t)=\min \left\{\left\{\begin{array}{cl}
\mathbf{f}_{i}\left(\boldsymbol{\rho}_{i}(t, 0-)\right) & \text { if } \boldsymbol{\rho}_{i}(t, 0) \leq \boldsymbol{\rho}_{i}^{\text {crit }} \\
\mathbf{f}_{i}^{\text {max }} & \text { if } \boldsymbol{\rho}_{i}(t, 0)>\boldsymbol{\rho}_{i}^{\text {crit }}
\end{array}\right\}, \mathbf{c}_{i}(t)\left(M-\sum_{j \in \mathcal{O}} \mathbf{q}_{j}(t)\right)\right\}
$$

This formulation takes into account the fact that the available space in the buffer might be limited and not sufficient to support the demand function. The minimum selects the inflow as the corresponding demand to the density on the entering link, except if it exceeds the capacity of the buffer, in which case it allocates for the boundary condition the space left inside. A similar approach was developed in [3, Definition 2.14]. We will prove later that the buffer never reaches capacity, i.e. the right-hand side of the minimum remains strictly positive over time. 


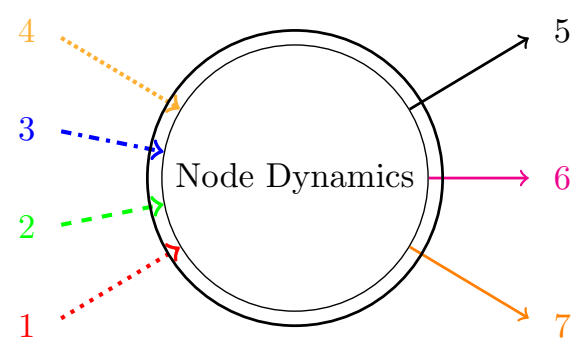

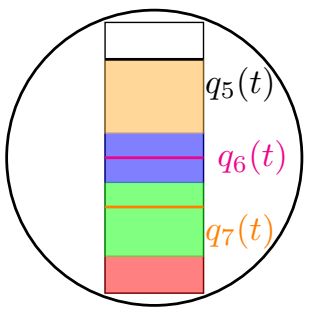

Section 2.3.1

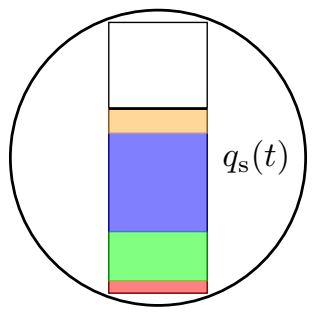

Section 2.3.2

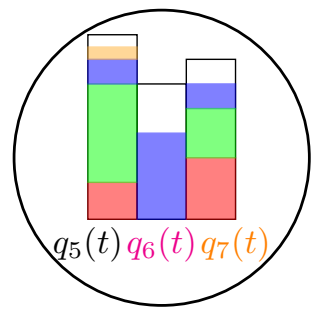

Section 2.3.3

FIG. 2.2. Illustration of the node/intersection dynamics. Left: the Single-Buffer/Multi-Queues model in Section 2.3.1, consisting in one common buffer shared by the (three) leaving queues. All incoming traffic has contributed to the buffer and only the buffer state is reported back to the incoming roads. Middle: the Single-Buffer/Single-Queues model in Section 2.3.2; Again, only one buffer exists but also only one queue is serving all leaving links simultaneously. Also here, only the buffer state is reported back to the incoming roads. Right: the Independent buffer model in Section 2.3.3 consisting in three different buffers serving three different queues corresponding to the three different leaving links. These buffers can have different sizes, and reports back to the incoming roads depending on their respective loads and routing functions.

In the same way as we have defined the maximum possible flux entering, we now define the maximum possible flux exiting, which corresponds to the supply function. Denoting by

$$
0 \leq \boldsymbol{\theta}_{i, j}(t) \leq 1 \quad t \in[0, T]
$$

the time-dependent fraction of vehicles traveling from $\operatorname{road} i \in \mathcal{I}$ into $j \in \mathcal{O}$ with $\sum_{j \in \mathcal{O}} \boldsymbol{\theta}_{i, j}(t)=1$, we set the exiting boundary condition as the minimum between the demand of traffic and the supply of space on the exit:

$$
\boldsymbol{\Gamma}_{j}(t)=\left\{\begin{array}{cl}
\left\{\begin{array}{cl}
\mathbf{f}_{j}\left(\boldsymbol{\rho}_{j}(t, 0+)\right) & \text { if } \boldsymbol{\rho}_{j}(t, 0)>\boldsymbol{\rho}_{j}^{\text {crit }} \\
\mathbf{f}_{j}^{\max } & \text { if } \boldsymbol{\rho}_{j}(t, 0) \leq \boldsymbol{\rho}_{j}^{\text {crit }}
\end{array}\right\} & \text { if } \mathbf{q}_{j}(t)>0, \\
\left.\min \left\{\begin{array}{cl}
\mathbf{f}_{j}\left(\boldsymbol{\rho}_{j}(t, 0+)\right) & \text { if } \boldsymbol{\rho}_{j}(t, 0)>\boldsymbol{\rho}_{j}^{\text {crit }} \\
\mathbf{f}_{j}^{\text {max }} & \text { if } \boldsymbol{\rho}_{j}(t, 0) \leq \boldsymbol{\rho}_{j}^{\text {crit }}
\end{array}\right\}, \sum_{i \in \mathcal{I}} \boldsymbol{\theta}_{i, j}(t) \boldsymbol{\Gamma}_{i}(t)\right\} & \text { if } \mathbf{q}_{j}(t)=0 .
\end{array}\right.
$$

For $t \in[0, T]$, if $\mathbf{q}_{j}(t)>0$ for $j \in \mathcal{O}$, vehicles are stored in the buffer and wait to access the outgoing road. Thus, the boundary condition is determined by the supply on the exit road. Either the traffic is in free flow and we can allocate $\mathbf{f}_{j}^{\max }$, or it is congested, and we can assign the flux corresponding to the traffic state on the specific road. If 
$\mathbf{q}_{j}(t)=0$, the incoming flow at the intersection can directly exit onto the outgoing road. Thus, the minimum assigns as a boundary condition the minimum between the supply and the demand.

Finally, we need to define the dynamics governing the buffer states with an additional equation accounting for the flow which enters and leaves the buffer at time $t \in[0, T]:$

$$
\mathbf{q}_{j}^{\prime}(t)=\sum_{i \in \mathcal{I}} \boldsymbol{\theta}_{i, j}(t) \boldsymbol{\Gamma}_{i}(t)-\boldsymbol{\Gamma}_{j}(t), \quad t \in[0, T], j \in \mathcal{O} .
$$

2.3.2. Single-Buffer/Single-Queue. In the Single-Buffer/Single-Queue scenario (see [21]), we do not assign one buffer per exiting link, but only define one scalar buffer state at the level of the intersection, $q_{\mathrm{s}}(t)$ for $t \in[0, T]$, serving all incoming and outgoing roads. The remaining space in the buffer at time $t$ is $M-q_{\mathrm{s}}(t)$ for $M \in \mathbb{R}_{>0}$

$$
\boldsymbol{\Gamma}_{i}(t)=\min \left\{\left\{\begin{array}{cl}
\mathbf{f}_{i}\left(\boldsymbol{\rho}_{i}(t, 0-)\right) & \text { if } \boldsymbol{\rho}_{i}(t, 0) \leq \boldsymbol{\rho}_{i}^{\text {crit }} \\
\mathbf{f}_{i}^{\text {max }} & \text { if } \boldsymbol{\rho}_{i}(t, 0)>\boldsymbol{\rho}_{i}^{\text {crit }}
\end{array}\right\}, \mathbf{c}_{i}(t)\left(M-q_{\mathrm{s}}(t)\right)\right\} .
$$

Denoting by $0 \leq \boldsymbol{\theta}_{j}(t) \leq 1$ the time-dependent fraction of vehicles traveling to the road $j \in \mathcal{O}$ with $\sum_{j \in \mathcal{O}} \boldsymbol{\theta}_{j}(t)=1$ (the turning ratio is here independent of the road from which the vehicles actually entered)

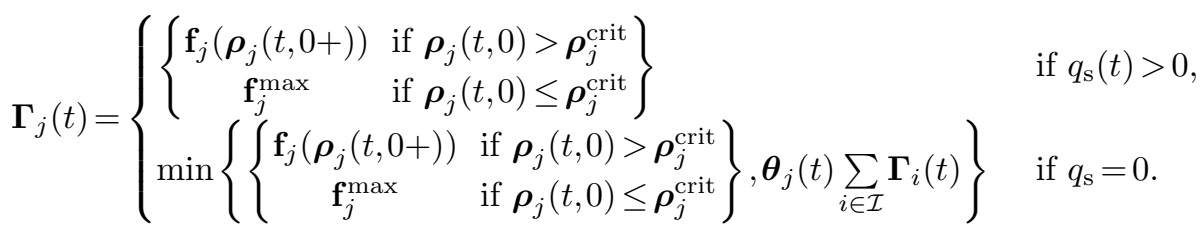

The dynamics for the queue are then:

$$
q_{\mathrm{s}}^{\prime}(t)=\sum_{i \in \mathcal{I}} \boldsymbol{\Gamma}_{i}(t)-\sum_{j \in \mathcal{O}} \boldsymbol{\Gamma}_{j}(t)
$$

2.3.3. Independent-Buffers. In this case, we allocate one buffer $\mathbf{M}_{j} \in \mathbb{R}_{>0}$ and one queue $\mathbf{q}_{j}(t)$ to each exit link $j \in \mathcal{O}$. The remaining space in each buffer is then $\mathbf{M}_{j}-\mathbf{q}_{j}(t), t \in[0, T]$, and $\boldsymbol{\theta}_{i, j}$ is the ratio of flow which is assigned from road $i \in \mathcal{I}$ to road $j \in \mathcal{O}$ satisfying conservation of flow, i.e. $\sum_{j \in \mathcal{O}} \boldsymbol{\theta}_{i, j}(t)=1,0 \leq \boldsymbol{\theta}_{i, j} \leq 1 \forall(i, j) \in \mathcal{I} \times \mathcal{O}$. We then obtain for the incoming roads the dynamics

$$
\boldsymbol{\Gamma}_{i}(t)=\min \left\{\left\{\begin{array}{cl}
\mathbf{f}_{i}\left(\boldsymbol{\rho}_{i}(t, 0-)\right) & \text { if } \boldsymbol{\rho}_{i}(t, 0) \leq \boldsymbol{\rho}_{i}^{\text {crit }} \\
\mathbf{f}_{i}^{\text {max }} & \text { if } \boldsymbol{\rho}_{i}(t, 0)>\boldsymbol{\rho}_{i}^{\text {crit }}
\end{array}\right\}, \min _{j \in \mathcal{O}} \frac{\mathbf{M}_{j}-\mathbf{q}_{j}(t)}{\boldsymbol{\theta}_{i, j}(t)}\right\}, i \in \mathcal{I}
$$

and for the outgoing links the boundary terms read for $j \in \mathcal{O}$ as

$$
\boldsymbol{\Gamma}_{j}(t)=\left\{\begin{array}{cl}
\left\{\begin{array}{cl}
\mathbf{f}_{j}\left(\boldsymbol{\rho}_{j}(t, 0+)\right) & \text { if } \boldsymbol{\rho}_{j}(t, 0)>\boldsymbol{\rho}_{j}^{\text {crit }} \\
\mathbf{f}_{j}^{\max } & \text { if } \boldsymbol{\rho}_{j}(t, 0) \leq \boldsymbol{\rho}_{j}^{\text {crit }}
\end{array}\right\} & \text { if } \mathbf{q}_{j}(t)>0, \\
\min \left\{\left\{\begin{array}{cl}
\mathbf{f}_{j}\left(\boldsymbol{\rho}_{j}(t, 0+)\right) & \text { if } \boldsymbol{\rho}_{j}(t, 0)>\boldsymbol{\rho}_{j}^{\text {crit }} \\
\mathbf{f}_{j}^{\text {max }} & \text { if } \boldsymbol{\rho}_{j}(t, 0) \leq \boldsymbol{\rho}_{j}^{\text {crit }}
\end{array}\right\}, \sum_{i \in \mathcal{I}} \boldsymbol{\theta}_{i, j}(t) \boldsymbol{\Gamma}_{i}(t)\right\} & \text { if } \mathbf{q}_{j}(t)=0 .
\end{array}\right.
$$

In the case $\boldsymbol{\theta}_{i, j}(t)=0$, we define $\frac{\mathbf{M}_{j}-\mathbf{q}_{j}(t)}{\boldsymbol{\theta}_{i, j}(t)}=+\infty$. The dynamics for the queues are stated as

$$
\mathbf{q}_{j}^{\prime}(t)=\sum_{i \in \mathcal{I}} \boldsymbol{\theta}_{i, j}(t) \boldsymbol{\Gamma}_{i}(t)-\boldsymbol{\Gamma}_{j}(t), \quad t \in[0, T], j \in \mathcal{O}
$$


with the bounds on the queues

$$
\mathbf{0} \leq \mathbf{q}_{j}(t) \leq \mathbf{M}_{j} \quad \forall t \in[0, T], j \in \mathcal{O} .
$$

2.4. Derivation of the corresponding Hamilton-Jacobi framework. In this section, we will show formally how to derive the proper Hamilton-Jacobi framework for the considered problem class, assuming smooth solutions of the conservation laws. We will only consider the Single-Buffer/Multi-Queues case, the other two cases can be derived the same way.

2.4.1. Number of vehicles exiting an entry link. On any incoming link $i \in \mathcal{I}$ the initial condition $\boldsymbol{\rho}_{0, i}^{\text {in }}$ on the level of conservation laws can be used to define the initial datum of the Hamilton-Jacobi formulation for $x \in \mathbb{R}_{<0}$ :

$$
\mathbf{V}_{0}^{\text {in }}(x)=\int_{-\infty}^{x} \boldsymbol{\rho}_{0}^{\text {in }}(y) \mathrm{d} y .
$$

$\mathbf{V}_{i}^{\text {in }}$ is the integrated variable of the density for $t \in[0, T]$ :

$$
\begin{aligned}
\partial_{x} \mathbf{V}_{i}^{\text {in }}(t, x) & =\boldsymbol{\rho}_{i}(t, x) \\
\mathbf{V}_{i}^{\text {in }}(t, x) & =\int_{-\infty}^{x} \boldsymbol{\rho}_{i}(t, y) \mathrm{d} y .
\end{aligned}
$$

The function $\mathbf{V}^{\text {in }}$ is in traffic simulation often called Moskowitz function (compare for instance $[13,28])$. We can then write the conservation of vehicles on $\mathbb{R}_{<0}$, with $\boldsymbol{N}_{i}(t)=\int_{0}^{t} \mathbf{f}_{i}\left(\boldsymbol{\rho}_{i}(s, 0)\right) \mathrm{d} s$ the total number of vehicles that have left the link $i \in \mathcal{I}$ at time $t \in(0, T)$ :

$$
\begin{aligned}
\frac{\mathrm{d}}{\mathrm{d} s} \int_{-\infty}^{0} \boldsymbol{\rho}_{i}(s, y) \mathrm{d} y & =-\mathbf{f}_{i}\left(\boldsymbol{\rho}_{i}(s, 0)\right) \\
\int_{0}^{t} \frac{\mathrm{d}}{\mathrm{d} s} \int_{-\infty}^{0} \boldsymbol{\rho}_{i}(s, y) \mathrm{d} y \mathrm{~d} s & =-\int_{0}^{t} \mathbf{f}_{i}\left(\boldsymbol{\rho}_{i}(s, 0)\right) \mathrm{d} s .
\end{aligned}
$$

Thus, we obtain for $t \in(0, T)$

$$
\boldsymbol{N}_{i}(t)=\mathbf{V}_{0, i}^{\mathrm{in}}(0)-\mathbf{V}_{i}^{\mathrm{in}}(t, 0) .
$$

2.4.2. Number of vehicles that have reached the intersection. $\boldsymbol{N}_{i}(t)$ represents the number of vehicles that reached the intersection during the time interval $[0, t]$. Among those vehicles, some want to access a given $\operatorname{road} j \in \mathcal{O}$. We name $\mathbf{F}_{j}(t)$ the number of vehicles that have reached the intersection before time $t$ and wish to turn into a given road $j \in \mathcal{O}$. We can then integrate and state for $t \in[0, T]$ :

$$
\begin{aligned}
\mathbf{F}_{j}(t) & =\mathbf{q}_{j}(0)+\sum_{i \in \mathcal{I}} \int_{0}^{t} \boldsymbol{\theta}_{i, j}(s) \boldsymbol{N}_{i}^{\prime}(s) d s \\
& =\mathbf{q}_{j}(0)+\sum_{i \in \mathcal{I}}\left[\boldsymbol{\theta}_{i, j}(s) \boldsymbol{N}_{i}(s)\right]_{0}^{t}-\sum_{i \in I} \int_{0}^{t} \boldsymbol{\theta}_{i, j}^{\prime}(s) \boldsymbol{N}_{i}(s) \mathrm{d} s \\
& =\mathbf{q}_{j}(0)+\sum_{i \in \mathcal{I}} \boldsymbol{\theta}_{i, j}(t) \boldsymbol{N}_{i}(t)-\sum_{i \in \mathcal{I}} \int_{0}^{t} \boldsymbol{\theta}_{i, j}^{\prime}(s) \boldsymbol{N}_{i}(s) \mathrm{d} s
\end{aligned}
$$




$$
=\mathbf{q}_{j}(0)+\sum_{i \in \mathcal{I}} \boldsymbol{\theta}_{i, j}(t)\left(\mathbf{V}_{0, i}^{\mathrm{in}}(0)-\mathbf{V}_{i}^{\mathrm{in}}(t, 0)\right)-\sum_{i \in \mathcal{I}} \int_{0}^{t} \boldsymbol{\theta}_{i, j}^{\prime}(s)\left(\mathbf{V}_{0, i}^{\mathrm{in}}(0)-\mathbf{V}_{i}^{\mathrm{in}}(s, 0)\right) \mathrm{d} s .
$$

This formulation on $\mathbf{F}$ will be used later to define the proper fixed-point mapping Section 4.2.

2.4.3. Cumulative vehicle count formulation - vehicles reaching a given exit $j \in \mathcal{O}$. Let us now apply the same reasoning for a given exit $j \in \mathcal{O}$. The initial condition reads as follows:

$$
\mathbf{V}_{0}^{\text {out }}(x)=\int_{0}^{x} \boldsymbol{\rho}_{0}^{\text {out }}(y) \mathrm{d} y, \quad x \in(0, \infty)
$$

$\mathbf{V}^{\text {out }}$ is the cumulative density so that we obtain for $(t, x) \in(0, T) \times \mathbb{R}_{>0}, j \in \mathcal{O}$

$$
\begin{aligned}
\partial_{x} \mathbf{V}_{j}^{\text {out }}(t, x) & =\boldsymbol{\rho}_{j}(t, x) \\
\mathbf{V}_{j}^{\text {out }}(t, x) & =\int_{0}^{x} \boldsymbol{\rho}_{j}(t, y) \mathrm{d} y+\boldsymbol{\beta}(t)
\end{aligned}
$$

with $\beta$ a function yet to be determined. $\mathbf{V}_{j}^{\text {out }}$ satisfies the following Hamilton-Jacobi equation:

$$
\begin{array}{r}
\partial_{t} \mathbf{V}_{j}^{\text {out }}(t, x)+\mathbf{f}_{j}\left(\partial_{x} \mathbf{V}_{j}^{\text {out }}(t, x)\right)=0 \\
\int_{0}^{x} \partial_{t} \boldsymbol{\rho}_{j}(t, y) \mathrm{d} y+\boldsymbol{\beta}^{\prime}(t)+\mathbf{f}_{j}\left(\boldsymbol{\rho}_{j}(t, x)\right)=0 .
\end{array}
$$

Plugging in $x=0$

$$
\boldsymbol{\beta}(t)+\int_{0}^{t} \mathbf{f}_{j}\left(\boldsymbol{\rho}_{j}(s, 0)\right) \mathrm{d} s=0
$$

we then obtain

$$
\mathbf{V}_{j}^{\text {out }}(t, x)=\int_{0}^{x} \boldsymbol{\rho}_{j}(t, y) \mathrm{d} y-\int_{0}^{t} \mathbf{f}_{j}\left(\boldsymbol{\rho}_{j}(s, 0)\right) \mathrm{d} s .
$$

We can then write the conservation of vehicles on any segment $[0, x]$ :

$$
\begin{aligned}
\frac{\mathrm{d}}{\mathrm{d} s} \int_{0}^{x} \boldsymbol{\rho}_{j}(s, y) \mathrm{d} y & =\mathbf{f}_{j}\left(\boldsymbol{\rho}_{j}(s, 0)\right)-\mathbf{f}_{j}\left(\boldsymbol{\rho}_{j}(s, x)\right) \\
\int_{0}^{t} \frac{\mathrm{d}}{\mathrm{d} s} \int_{0}^{x} \boldsymbol{\rho}_{j}(s, y) \mathrm{d} y \mathrm{~d} s & =\int_{0}^{t}\left[\mathbf{f}_{j}\left(\boldsymbol{\rho}_{j}(s, 0)\right)-\mathbf{f}_{j}\left(\boldsymbol{\rho}_{j}(s, x)\right)\right] \mathrm{d} s \\
\int_{0}^{x} \boldsymbol{\rho}_{j}(t, y) \mathrm{d} y-\int_{0}^{x} \boldsymbol{\rho}_{j}(0, y) \mathrm{d} y & =\int_{0}^{t}\left[\mathbf{f}_{j}\left(\boldsymbol{\rho}_{j}(s, 0)\right)-\mathbf{f}_{j}\left(\boldsymbol{\rho}_{j}(s, x)\right)\right] \mathrm{d} s \\
\mathbf{V}_{j}^{\text {out }}(t, x)+\int_{0}^{t} \mathbf{f}_{j}\left(\boldsymbol{\rho}_{j}(s, 0)\right) \mathrm{d} s-\mathbf{V}_{0, j}^{\text {out }}(x) & =\int_{0}^{t} \mathbf{f}_{j}\left(\boldsymbol{\rho}_{j}(s, 0)\right) \mathrm{d} s-\int_{0}^{t} \mathbf{f}_{j}\left(\boldsymbol{\rho}_{j}(s, x)\right) \mathrm{d} s \\
\mathbf{V}_{0, j}^{\text {out }}(x)-\mathbf{V}_{j}^{\text {out }}(t, x) & =\int_{0}^{t} \mathbf{f}_{j}\left(\boldsymbol{\rho}_{j}(s, x)\right) \mathrm{d} s .
\end{aligned}
$$

Thus the number $\mathbf{V}_{0, j}^{\text {out }}(x)-\mathbf{V}_{j}^{\text {out }}(t, x)$ represents the number of vehicles that have crossed the location $x \in \mathbb{R}_{>0}$ during the interval $[0, t]$. In particular, if we call $\boldsymbol{S}_{j}$ the total number of vehicles that have entered the link $j \in \mathcal{O}$ at time $t \in[0, T]$,

$$
\boldsymbol{S}_{j}(t)=-\mathbf{V}_{j}^{\text {out }}(t, 0) .
$$


2.4.4. Queue length. As a direct consequence of the conservation of mass, the buffer will store the difference between the number of vehicles that wanted to access $j \in \mathcal{O}$ and the number of vehicles that actually entered $j \in \mathcal{O}$. Thus, the length of the queue at the entrance of $j$ is given by:

$$
\mathbf{q}_{j}(t)=\mathbf{F}_{j}(t)-\boldsymbol{S}_{j}(t), t \in[0, T]
$$

REMARK 2.1. We will prove later in Lemma 4.7 that the buffer can never exceed the prescribed capacity $\mathbf{M}$, making the model reasonable. This is due to the dynamics which cause a natural spill-back when the buffer gets close to capacity so that less vehicles can actually enter as flow into the buffer is decreased.

\section{Definition of Solutions}

As a solution on a given junction in the conservation law framework we define:

DeFinition 3.1 (Definition of the solution of the system of conservation laws and buffers). We consider a junction with $i \in \mathcal{I}$ incoming links and $j \in \mathcal{O}$ outgoing links. We assume in addition that Assumption 2.1 is satisfied and initial data $\boldsymbol{\rho}_{0, i} \in L^{1}\left(\mathbb{R}_{<0}\right) \cap$ $L^{\infty}\left(\mathbb{R}_{<0}\right)$ with $0 \leq \boldsymbol{\rho}_{0, i} \leq \boldsymbol{\rho}_{i}^{\max }$ and $\boldsymbol{\rho}_{0, j} \in L^{1}\left(\mathbb{R}_{>0}\right) \cap L^{\infty}\left(\mathbb{R}_{>0}\right)$ with $0 \leq \boldsymbol{\rho}_{0, j} \leq \boldsymbol{\rho}_{j}^{\max }$ be given. A solution to the initial boundary value problem with buffers as in Section 2 is given iff:

(1) $\boldsymbol{\rho}^{\text {in }}, \boldsymbol{\rho}^{\text {out }}$ are weak entropy solutions of the conservation laws at the entering and exiting links and satisfy

$$
\boldsymbol{\rho}_{i}^{\text {in }} \in C\left([0, T] ; L^{1}\left(\mathbb{R}_{\leq 0}\right)\right), i \in \mathcal{I} \quad \boldsymbol{\rho}_{j}^{\text {out }} \in C\left([0, T] ; L^{1}\left(\mathbb{R}_{\geq 0}\right)\right), j \in \mathcal{O} .
$$

(2) For the three different types of queues with buffer as stated in Section 2.3 we have

- Single-Buffer/Multi-Queues: For a given $M \in \mathbb{R}_{>0}$ the queues $\mathbf{q}$ are determined by Eq. (2.6) for a given split ratio $\boldsymbol{\theta}_{i, j} \in B V((0, T))$ satisfying

$$
0 \leq \boldsymbol{\theta}_{i, j}(t) \leq 1, \quad \sum_{j \in \mathcal{O}} \boldsymbol{\theta}_{i, j}(t)=1 \forall t \in[0, T] \text { a.e. } \forall(i, j) \in \mathcal{I} \times \mathcal{O} .
$$

- Single-Buffer/Single-Queue: For a given $M \in \mathbb{R}_{>0}$, the queue $q_{s}$ is determined by Eq. (2.7) for a given split ratio $\boldsymbol{\theta}_{j} \in B V((0, T))$ satisfying

$$
0 \leq \boldsymbol{\theta}_{j}(t) \leq 1, \quad \sum_{j \in \mathcal{O}} \boldsymbol{\theta}_{j}(t)=1 \forall t \in[0, T] \text { a.e. } j \in \mathcal{O} .
$$

- Independent-Buffers: For given $\mathbf{M} \in \mathbb{R}_{>0}^{|\mathcal{O}|}$ the queues $\mathbf{q}$ are determined by $E q$. (2.9) for a given split ratio $\boldsymbol{\theta}_{i, j} \in B V((0, T))$ satisfying

$$
0 \leq \boldsymbol{\theta}_{i, j}(t) \leq 1, \quad \sum_{j \in \mathcal{O}} \boldsymbol{\theta}_{i, j}(t)=1 \forall t \in[0, T] \text { a.e. } \forall(i, j) \in \mathcal{I} \times \mathcal{O} .
$$

(3) For the three different types of queues with buffer the boundary conditions on any incoming link $i \in \mathcal{I}$ and any outgoing link $j \in \mathcal{O}$ are respectively given by

- Single-Buffer/Multi-Queues: Section 2.3.1,

- Single-Buffer/Single-Queue: Section 2.3.2,

- Independent-Buffers: Section 2.3.3.

In the stated initial boundary values problems in $\boldsymbol{\rho}$, the boundary datum is prescribed in the sense of Bardos-Leroux-Nédélec [7], see [16]. 


\section{Existence/Uniqueness of Solutions - Hamilton-Jacobi Framework}

In the following, we will provide mathematical results for the Single-Buffer/MultiQueues, the results for the Single-Buffer/Single-Queue and the Independent-Buffers can be derived the same way.

\subsection{Basic results and Properties of Hamilton-Jacobi Equations.}

Definition 4.1 (The Legendre-Fenchel transform). Suppose we have a flux function $f \in C^{2}\left(\left[0, \rho^{\max }\right] ; \mathbb{R}_{>0}\right)$, strictly concave with $\rho^{\max } \in \mathbb{R}_{>0}$ given. Then, we define the Legendre-Fenchel transform $f^{*}$ of $f$ as

$$
f^{*}(x):=\inf _{u \in\left[0, \rho^{\max }\right]}\{u x-f(u)\}, x \in \operatorname{Dom}\left(f^{*}\right) .
$$

Thereby, the domain for the Legendre transform $f^{*}$ is defined as

$$
\operatorname{Dom}\left(f^{*}\right):=\left\{x^{*} \in \mathbb{R}: \inf _{x \in\left[0, \rho^{\max }\right]}\left\{x x^{*}-f(x)\right\}<\infty\right\}=\mathbb{R} .
$$

Lemma 4.1 (Properties of the Legendre-Fenchel transform). Let the Legendre-Fenchel transform $f^{*}$ as defined in Definition 4.1 be given. Then, the following properties hold:

(1) $f^{*}$ is Lipschitz-continuous, i.e. $\left|f^{*}(x)-f^{*}(y)\right| \leq \rho^{\max }|x-y| \forall x, y \in \operatorname{Dom}\left(f^{*}\right)$.

(2) $f^{*}$ is concave with $\operatorname{Dom}\left(f^{*}\right)=\mathbb{R}$.

(3) The infimum in the definition of $f^{*}$ is attained, i.e.

$$
\inf _{u \in\left[0, \rho^{\max }\right]}\{u x-f(u)\}=\min _{u \in\left[0, \rho^{\max }\right]}\{u x-f(u)\} .
$$

(4) $f^{*}$ is bounded from above, i.e. $\max _{x \in \mathbb{R}} f^{*}(x) \leq-f(0)=0$.

(5) The dual of $f^{*}$ is $f$ in the following meaning: $\forall x \in\left[0, \rho^{\max }\right]$ the following equality holds $f(x)=\inf _{u \in \mathbb{R}}\left\{u x-f^{*}(u)\right\}$.

Proof.

(1) Let $x, y \in \operatorname{Dom}\left(f^{*}\right)$ and let $v^{*} \in[0,1]$ denote the point where the infimum is reached for the expression $f^{*}(y)$. Then, we obtain

$$
\begin{aligned}
f^{*}(x)-f^{*}(y) & =\inf _{u \in\left[0, \rho^{\max }\right]}\{u x-f(u)\}-\inf _{v \in\left[0, \rho^{\max }\right]}\{v y-f(v)\} \\
& \leq v^{*} x-f\left(v^{*}\right)-v^{*} y+f\left(v^{*}\right) \leq v^{*}(x-y) \leq v^{*}|x-y| \leq \rho^{\max }|x-y| .
\end{aligned}
$$

By reverting the role of $x$ and $y$, we conclude that $f^{*}$ is Lipschitz-continuous with Lipschitz-constant $\rho^{\max }$.

$(2),(3),(4)$ We do not detail the proof here.

(5) See $[8]$.

REMARK 4.1 (Flux function regularity). Assumption 2.1 and also the regularity proposed for defining the Legendre-Fenchel transform in Section 4.1 can be weakened to $f \in C^{1}(\mathbb{R})$, strictly concave with super-linear growth. However, in the framework of traffic flow modelling, the additional regularity $f \in C^{2}(\mathbb{R})$ is not too restrictive and is aligned with [9-11], on which this contribution is based on.

For the following results it becomes mandatory to present a theorem for one-sided boundary datum and initial value of Hamilton-Jacobi equations. The following theorem not only gives an explicit solution formula in terms of a minimization problem, it also states the relation of the solution formula to the corresponding conservation law with one-sided boundary datum. 
THEOREM 4.1 (Explicit solution of HJ-Equations, relation to the conservation law [32]). Consider the following HJ Equation:

$$
\begin{aligned}
\partial_{t} \tilde{v}(t, x)+g\left(\partial_{x} \tilde{v}(t, x)\right) & =0 & & (t, x) \in(0, T) \times \mathbb{R}_{>0} \\
\tilde{v}(0, x) & =\tilde{v}_{0}(x) & & x \in \mathbb{R}_{>0} \\
\partial_{x} \tilde{v}(t, x=0) & =\bar{\rho}_{b}(t) & & t \in(0, T)
\end{aligned}
$$

with $g \in C^{2}(\mathbb{R} ; \mathbb{R})$ being strictly convex, satisfying $\lim _{y \rightarrow \pm \infty} \frac{g(y)}{|y|}=\infty$ and $\rho_{b} \in L^{\infty}((0, T))$ given with $\bar{\rho}_{b}(t)=\max \left\{\rho_{b}(t), \lambda\right\}$ and $\lambda$ be implicitly defined as $g(\lambda)=\min _{u \in \mathbb{R}} g(u)$ as well as $\rho_{0} \in L^{\infty}\left(\mathbb{R}_{>0}\right) \cap L^{1}\left(\mathbb{R}_{>0}\right)$ with $v_{0}(x) \equiv \int_{0}^{x} \rho_{0}(y) \mathrm{d} y, x \in \mathbb{R}_{>0}$. Then, the solution of the Hamilton-Jacobi equation can be stated in terms of a minimization problem involving boundary and initial terms and reads for $(t, x) \in[0, T] \times \mathbb{R}_{>0}$ as

$$
\begin{aligned}
& \tilde{v}(t, x)=\min \left\{\min _{y \in \mathbb{R}_{\geq 0}}\left\{t g^{*}\left(\frac{x-y}{t}\right)+\tilde{v}_{0}(y)\right\},\right. \\
&\left.\min _{\substack{0 \leq t_{2} \leq t_{1} \leq t \\
y \in \mathbb{R}_{\geq 0}}}\left\{\tilde{v}_{0}(y)+g^{*}\left(\frac{-y}{t_{2}}\right) t_{2}+\left(t-t_{1}\right) g^{*}\left(\frac{x}{t-t_{1}}\right)-\int_{t_{2}}^{t_{1}} g\left(\bar{\rho}_{b}(\theta)\right) \mathrm{d} \theta\right\}\right\}
\end{aligned}
$$

where

$$
g^{*}(x):=\sup _{u \in \mathbb{R}}\{u x-g(u)\}
$$

In addition, $\tilde{v} \in \operatorname{Lip}\left([0, T] \times \mathbb{R}_{\geq_{0}}\right)$ and the partial derivative of $v$ with respect to the spatial variable is the weak entropy solution of the corresponding conservation law satisfying the boundary condition in the sense of Bardos-Leroux-Nédélec [7] :

$$
\begin{aligned}
\partial_{t} \rho(t, x)+\partial_{x} g(\rho(t, x)) & =0 & & (t, x) \in(0, T) \times \mathbb{R}_{\geq 0} \\
\rho(0, x) & =\rho_{0}(x) & & x \in \mathbb{R}_{\geq 0} \\
\rho(t, 0) & =\bar{\rho}_{b}(t) & & t \in(0, T) .
\end{aligned}
$$

Proof. The proof can be found in [32].

LEMMA 4.2 (Solution formula for the Hamilton-Jacobi equation related to Eqs. (2.1) to (2.4)). Let $\rho_{0} \in L^{1}\left(\mathbb{R}_{<0}\right) \cap L^{\infty}\left(\mathbb{R}_{<0}\right)$ with $0 \leq \rho_{0}(x) \leq \rho^{\max }, x \in \mathbb{R}_{<0}$ a.e. be given. Assume the flux function $f \in C^{2}(\mathbb{R} ; \mathbb{R})$ being strictly concave and $\lim _{y \rightarrow \pm \infty} \frac{f(y)}{|y|}=-\infty$. Consider the following conservation law on the half plane:

$$
\begin{aligned}
\partial_{t} \rho(t, x)+\partial_{x} f(\rho(t, x)) & =0 & & (t, x) \in(0, T) \times \mathbb{R}_{<0} \\
\rho(0, x) & =\rho_{0}(x) & & x \in \mathbb{R}_{<0} \\
f(\rho(t, 0)) & =h(t) & & t \in(0, T)
\end{aligned}
$$

with $h \in L^{\infty}((0, T))$ and $0 \leq h(t) \leq f^{\max }, t \in(0, T)$ a.e. be given. Then, the solution of the associated Hamilton-Jacobi equation can be stated in terms of a maximization problem involving boundary and initial term and reads for $(t, x) \in[0, T] \times \mathbb{R}_{\geq 0}$ as

$$
v(t, x)=\max \left\{\max _{y \in \mathbb{R} \leq 0}\left\{t f^{*}\left(\frac{x-y}{t}\right)+v_{0}(y)\right\},\right.
$$




$$
\left.\max _{\substack{0 \leq t_{2} \leq t_{1} \leq t \\ y \in \mathbb{R}_{\leq 0}}}\left\{v_{0}(y)+f^{*}\left(\frac{-y}{t_{2}}\right) t_{2}+\left(t-t_{1}\right) f^{*}\left(\frac{x}{t-t_{1}}\right)-\int_{t_{2}}^{t_{1}} h(\theta) \mathrm{d} \theta\right\}\right\} .
$$

Proof. We define $\bar{\rho}_{\mathrm{b}}(t)$ as the solution of $f\left(\bar{\rho}_{\mathrm{b}}(t)\right)=h(t)$ such that $\bar{\rho}_{\mathrm{b}}(t) \in\left[\rho^{\text {crit }}, \rho^{\max }\right]$. Consider the strictly convex function $g$ such that $g \equiv-f$. Given $x \in \mathbb{R}$, the following identity holds

$$
f^{*}(x)=\inf _{y \in \mathbb{R}}\{y x-f(y)\}=-\sup _{y \in \mathbb{R}}\{-y x+f(y)\}=-\sup _{y \in \mathbb{R}}\{-y x-g(y)\}=-g^{*}(-x) .
$$

Then we can apply Theorem 4.1 and obtain the solution to the following Initial Boundary Value Problem (IBVP)

$$
\begin{aligned}
\partial_{t} \tilde{v}(t, x)+g\left(\partial_{x} \tilde{v}(t, x)\right) & =0 & & (t, x) \in(0, T) \times \mathbb{R}_{\geq 0} \\
\tilde{v}(0, x) & =\tilde{v}_{0}(x) & & x \in \mathbb{R}_{\geq 0} \\
\partial_{x} \tilde{v}(t, x=0) & =\bar{\rho}_{\mathrm{b}}(t) & & t \in(0, T)
\end{aligned}
$$

for $(t, x) \in[0, T] \times \mathbb{R}_{\geq 0}$

$$
\begin{aligned}
\tilde{v}(t, x)=\min & \left\{\min _{y \in \mathbb{R}_{\geq 0}}\left\{g^{*}\left(\frac{x-y}{t}\right)+\tilde{v}_{0}(y)\right\},\right. \\
& \left.\min _{\substack{0 \leq t_{2} \leq t_{1} \leq t \\
y \in \mathbb{R}_{\geq 0}}}\left\{\tilde{v}_{0}(y)+g^{*}\left(\frac{-y}{t_{2}}\right) t_{2}+\left(t-t_{1}\right) g^{*}\left(\frac{x}{t-t_{1}}\right)-\int_{t_{2}}^{t_{1}} g\left(\bar{\rho}_{\mathrm{b}}(\theta)\right) \mathrm{d} \theta\right\}\right\} .
\end{aligned}
$$

For $x \in \mathbb{R}_{\leq 0}$ define $v(t, x)=-\tilde{v}(t,-x)$. Then $v$ is a solution of

$$
\begin{aligned}
-\partial_{t} v(t, x)+g\left(\partial_{x} v(t, x)\right) & =0 & & (t, x) \in(0, T) \times \mathbb{R}_{\leq 0} \\
v(0, x) & =-\tilde{v}_{0}(-x) & & x \in \mathbb{R}_{\leq 0} \\
\partial_{x} v(t, x=0) & =\bar{\rho}_{\mathrm{b}}(t) & & t \in(0, T)
\end{aligned}
$$

which is, by construction, the solution of

$$
\begin{aligned}
\partial_{t} v(t, x)+f\left(\partial_{x} v(t, x)\right) & =0 & & (t, x) \in(0, T) \times \mathbb{R}_{\leq 0} \\
v(0, x) & =-\tilde{v}_{0}(-x)=v_{0}(x) & & x \in \mathbb{R}_{\leq 0} \\
\partial_{x} v(t, x=0) & =\bar{\rho}_{\mathrm{b}}(t) & & t \in(0, T) .
\end{aligned}
$$

This is detailed in the following manipulations. Let $(t, x) \in[0, T] \times \mathbb{R}_{\geq 0}$ be given, we have

$$
\begin{aligned}
v(t, x)= & -\tilde{v}(t,-x) \\
= & -\min \left\{\min _{y \in \mathbb{R}_{\geq 0}}\left\{t g^{*}\left(\frac{-x-y}{t}\right)+\tilde{v}_{0}(y)\right\},\right. \\
& \left.\min _{\substack{0 \leq t_{2} \leq t_{1} \leq t \\
y \in \mathbb{R}_{\geq 0}}}\left\{\tilde{v}_{0}(y)+g^{*}\left(\frac{-y}{t_{2}}\right) t_{2}+\left(t-t_{1}\right) g^{*}\left(\frac{-x}{t-t_{1}}\right)-\int_{t_{2}}^{t_{1}} g\left(\bar{\rho}_{\mathrm{b}}(\theta)\right) \mathrm{d} \theta\right\}\right\} \\
= & \max \left\{\max _{y \in \mathbb{R}_{\geq 0}}\left\{-t g^{*}\left(\frac{-x-y}{t}\right)-\tilde{v}_{0}(y)\right\},\right.
\end{aligned}
$$




$$
\begin{aligned}
&\left.\max _{\substack{0 \leq t_{2} \leq t_{1} \leq t \\
y \in \mathbb{R}_{\geq 0}}}\left\{-\tilde{v}_{0}(y)-g^{*}\left(\frac{-y}{t_{2}}\right) t_{2}-\left(t-t_{1}\right) g^{*}\left(\frac{-x}{t-t_{1}}\right)+\int_{t_{2}}^{t_{1}} g\left(\bar{\rho}_{\mathrm{b}}(\theta)\right) \mathrm{d} \theta\right\}\right\} \\
&= \max \left\{\max _{y \in \mathbb{R}_{\geq 0}}\left\{t f^{*}\left(\frac{x+y}{t}\right)-\tilde{v}_{0}(y)\right\},\right. \\
&\left.\max _{\substack{0 \leq t_{2} \leq t_{1} \leq t \\
y \in \mathbb{R}_{\geq 0}}}\left\{-\tilde{v}_{0}(y)+f^{*}\left(\frac{y}{t_{2}}\right) t_{2}+\left(t-t_{1}\right) f^{*}\left(\frac{x}{t-t_{1}}\right)+\int_{t_{2}}^{t_{1}} g\left(\bar{\rho}_{\mathrm{b}}(\theta)\right) \mathrm{d} \theta\right\}\right\} \\
&=\max \left\{\max _{y \in \mathbb{R}_{\leq 0}}\left\{t f^{*}\left(\frac{x-y}{t}\right)+v_{0}(y)\right\},\right. \\
&\left.\max _{\substack{0 \leq t_{2} \leq t_{1} \leq t \\
y \in \mathbb{R}_{\leq 0}}}\left\{v_{0}(y)+f^{*}\left(\frac{-y}{t_{2}}\right) t_{2}+\left(t-t_{1}\right) f^{*}\left(\frac{x}{t-t_{1}}\right)-\int_{t_{2}}^{t_{1}} f\left(\bar{\rho}_{\mathrm{b}}(\theta)\right) \mathrm{d} \theta\right\}\right\}
\end{aligned}
$$

which is indeed the claimed formula.

REMARK 4.2. We note that in [32] the supremum in the Legendre Fenchel transform is selected on all $\mathbb{R}$ and not on a compact subset. In the present case, we restrict ourselves to $\left[0, \rho^{\max }\right]$ since the initial density is chosen in $\left[0, \rho^{\max }\right]$ and then the characteristics in the conservation law setting can only travel with speeds contained in $\left[f^{\prime}\left(\rho^{\max }\right), f^{\prime}(0)\right]$.

LEmma 4.3 (Semi-group property of the formula presented in Theorem 4.1). Given the formula for $v$ in Theorem 4.1, it satisfies the semi-group property, i.e. $\forall x \in \mathbb{R}_{\geq 0}, \forall t, \tilde{t} \in$ $[0, T], t>\tilde{t}$

$$
\begin{aligned}
& v(t, x)=\min \left\{\min _{y \in \mathbb{R}_{\geq 0}}\left\{(t-\tilde{t}) f^{*}\left(\frac{x-y}{t-\tilde{t}}\right)+v(\tilde{t}, y)\right\},\right. \\
&\left.\min _{\substack{\tilde{t} \leq t_{2} \leq t_{1} \leq t \\
y \in \mathbb{R}_{\geq 0}}}\left\{v(\tilde{t}, y)+f^{*}\left(\frac{-y}{t_{2}}\right) t_{2}+\left(t-t_{1}\right) f^{*}\left(\frac{x}{t-t_{1}}\right)-\int_{t_{2}}^{t_{1}} f\left(\bar{\rho}_{b}(\theta)\right) \mathrm{d} \theta\right\}\right\} .
\end{aligned}
$$

Proof. We refer the reader to [19] for the proof for the Cauchy problem. The extension with boundary datum can be derived similarly.

THEOREM 4.2 (Solution of the H.J.-Equations related to c.l. on the quarter plane). Let $F \in \operatorname{Lip}\left([0, T] ; \mathbb{R}_{\geq 0}\right)$ be given and consider for $T \in \mathbb{R}_{>0}$ and $\rho_{0} \in B V\left(\mathbb{R}_{\geq 0}\right)$ the initial boundary value problem

$$
\begin{aligned}
\partial_{t} \rho(t, x)+\partial_{x} f(\rho(t, x)) & =0 & & (t, x) \in(0, T) \times \mathbb{R}_{\geq 0} \\
\rho(0, x) & =\rho_{0}(x) & & x \in \mathbb{R}_{\geq 0}
\end{aligned}
$$

supplemented by the boundary datum at $x=0$ for $t \in(0, T)$ as

$$
f(\rho(t, 0))=b(t)
$$

where $b(t)$ is defined as

$$
b(t):= \begin{cases} \begin{cases}f^{\max } & \text { if } \rho(t, 0) \leq \rho^{\mathrm{crit}} \\
f(\rho(t, 0)) & \text { if } \rho(t, 0)>\rho^{\mathrm{crit}}\end{cases} & \text { if } q(t)>0 \\
\min \left\{\left\{\begin{array}{ll}
f^{\max } & \text { if } \rho(t, 0) \leq \rho^{\mathrm{crit}} \\
f(\rho(t, 0)) & \text { if } \rho(t, 0)>\rho^{\text {crit }}
\end{array}, F^{\prime}(t)\right\}\right. & \text { if } q(t)=0\end{cases}
$$


and $q \in W^{1, \infty}\left((0, T) ; \mathbb{R}_{\geq 0}\right)$ be given. Then, the correspondent Hamilton-Jacobi equation in $v$ reads as

$$
\begin{aligned}
\partial_{t} v(t, x)+f\left(\partial_{x} v(t, x)\right) & =0 & & (t, x) \in(0, T \\
v(0, x) & =\int_{0}^{x} \rho_{0}(y) \mathrm{d} y=v_{0}(x) & & x \in \mathbb{R}_{\geq 0} \\
f\left(\partial_{x} v(t, 0)\right) & =b(t) & & t \in(0, T)
\end{aligned}
$$

and obeys the explicit solution formula in terms of a minimization for $(t, x) \in(0, T) \times \mathbb{R}_{\geq 0}$

$$
v(t, x)=\max \left\{\max _{y \in \mathbb{R}_{\geq 0}}\left\{v_{0}(y)+t f^{*}\left(\frac{x-y}{t}\right)\right\}, \max _{0 \leq t_{1} \leq t}\left\{-F\left(t_{1}\right)+\left(t-t_{1}\right) f^{*}\left(\frac{x}{t-t_{1}}\right)\right\}\right\} .
$$

Moreover, the solution $v$ is Lipschitz-continuous and its spatial derivative provides the weak entropy solution of the conservation law with boundary data in the sense of BardosLeroux-Nédélec [7].

Proof. The proof can be found in [9, Section 8].

REMARK 4.1 (The mapping $F$ and $\mathbf{F}_{j}$ ). The function $F(t), t \in[0, T]$, will correspond in our framework to $\mathbf{F}_{j}, j \in \mathcal{O}$, the number of vehicles that reached the intersection at time $t$ and want to access exit $j$.

DEFinition 4.2 (Definition of initial data for the Hamilton-Jacobi equations). Given the initial datum for the conservation laws in Definition 3.1, we define the initial datum for the Hamilton-Jacobi equations as

- $\mathbf{V}_{0, i}^{\text {in }}(x):=\int_{-\infty}^{x} \boldsymbol{\rho}_{0, i}(z) \mathrm{d} z, x \in \mathbb{R}_{\leq 0}, i \in \mathcal{I} \quad \bullet \mathbf{V}_{0, j}^{\text {out }}(x):=\int_{0}^{x} \boldsymbol{\rho}_{0, j}(z) \mathrm{d} z, x \in \mathbb{R}_{\geq 0} j \in \mathcal{O}$.

4.2. The fixed-point problem. As formally shown in Section 2 we can pose the considered problem as a fixed-point problem on the level of Hamilton-Jacobi PDEs. Thereby, the buffer/queue in Section 2.3 is coupled to the boundary datum of the incoming and outgoing fluxes and the fluxes again by the state of the buffer/queue. This all will be made rigorous in this section:

Definition 4.3 (The decomposition of the fixed-point mapping). We define the following mappings:

- Let the priority function $\mathbf{c}_{i} \in L^{\infty}\left((0, T) ; \mathbb{R}_{\geq 0}\right)$ and $\mathbf{f}_{i}^{\max } \in \mathbb{R}_{>0}$ for $i \in \mathcal{I}$ be given. Then, we define the mapping

$$
\mathbf{h}: \begin{cases}\operatorname{Lip}\left([0, T] ; \mathbb{R}^{|\mathcal{O}|}\right) & \rightarrow \operatorname{Lip}\left([0, T] ; \mathbb{R}^{|\mathcal{I}|}\right) \\ \mathbf{q} & \mapsto\left(t \mapsto \min \left\{\mathbf{f}^{\max }, \mathbf{c}(t) \cdot\left(M-\sum_{j \in \mathcal{O}} \mathbf{q}_{j}(t)\right)\right\}\right)\end{cases}
$$

Thereby, the min is meant component-wise.

- For $i \in \mathcal{I}$ given initial datum $\mathbf{V}_{0}^{\text {in }}$ as defined in Definition 4.2 and $\mathbf{f}_{i}^{\max } \in \mathbb{R}_{>0}$ with $X:=\operatorname{Lip}([0, T])$ :

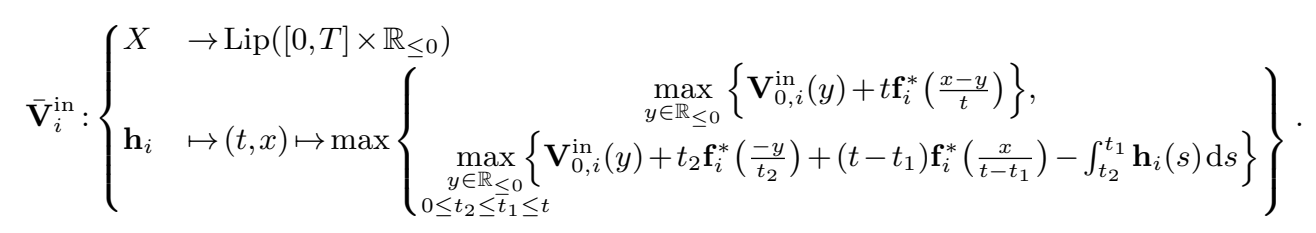


In addition, we define the solution evaluated at $x=0, \mathbf{V}^{\text {in }} \equiv \overline{\mathbf{V}}^{\text {in }}(\cdot, 0)$ on $[0, T]$ and $X:=\operatorname{Lip}([0, T])$

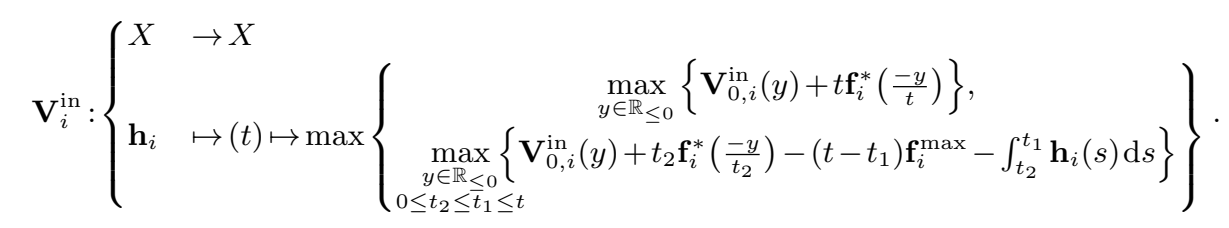

- Let $\mathbf{q}^{0} \in \mathbb{R}_{\geq 0}^{|\mathcal{O}|}$ with $\left\|\mathbf{q}^{0}\right\|_{1} \leq M$ and $\boldsymbol{\theta}$ as in Section 3 and Item 2, we define

$$
\mathbf{F}: \begin{cases}\operatorname{Lip}\left([0, T] ; \mathbb{R}^{|\mathcal{I}|}\right) & \mapsto \operatorname{Lip}\left([0, T] ; \mathbb{R}^{|\mathcal{O}|}\right) \\ \mathbf{V}^{\text {in }} & \mapsto\left(t \mapsto \mathbf{q}^{0}-\sum_{i \in I} \int_{0}^{t} \boldsymbol{\theta}_{i}(s) \frac{\mathrm{d}}{\mathrm{d} s} \mathbf{V}_{i}^{\mathrm{in}}(s) \mathrm{d} s\right)\end{cases}
$$

- Let $\mathbf{V}_{0}^{\text {out }}$ as defined in Definition 4.2 be given and $j \in \mathcal{O}$ we define for $X:=\operatorname{Lip}([0, T])$ :

$$
\overline{\mathbf{V}}_{j}^{\text {out }}: \begin{cases}X & \rightarrow \operatorname{Lip}\left([0, T] \times \mathbb{R}_{\geq 0}\right) \\ \mathbf{F}_{j} & \mapsto(t, x) \mapsto \max \left\{\max _{y \in \mathbb{R} \geq 0}\left\{\mathbf{V}_{0, j}^{\text {out }}(y)+t \mathbf{f}_{j}^{*}\left(\frac{x-y}{t}\right)\right\}, \max _{0 \leq t_{1} \leq t}\left\{-\mathbf{F}_{j}\left(t_{1}\right)+\left(t-t_{1}\right) \mathbf{f}_{j}^{*}\left(\frac{x}{t-t_{1}}\right)\right\}\right\} .\end{cases}
$$

In addition, we define the solution evaluated at $x=0, \mathbf{V}^{\text {out }} \equiv \overline{\mathbf{V}}^{\text {out }}(\cdot, 0)$ on $[0, T]$ :

$$
\mathbf{V}_{j}^{\text {out }}: \begin{cases}X & \rightarrow X \\ \mathbf{F}_{j} & \mapsto(t) \mapsto \max \left\{\max _{y \in \mathbb{R}_{\geq 0}}\left\{\mathbf{V}_{0, j}^{\text {out }}(y)+t \mathbf{f}_{j}^{*}\left(\frac{-y}{t}\right)\right\}, \max _{0 \leq t_{1} \leq t}\left\{-\mathbf{F}_{j}\left(t_{1}\right)-\left(t-t_{1}\right) \mathbf{f}_{j}^{\text {max }}\right\}\right\} .\end{cases}
$$

- Finally, we define

$$
\boldsymbol{\Lambda}: \begin{cases}\operatorname{Lip}\left([0, T] ; \mathbb{R}^{|\mathcal{O}|}\right) \times \operatorname{Lip}\left([0, T] ; \mathbb{R}^{|\mathcal{O}|}\right) & \rightarrow \operatorname{Lip}\left([0, T] ; \mathbb{R}^{|\mathcal{O}|}\right) \\ \left(\mathbf{F}, \mathbf{V}^{\text {out }}\right) & \mapsto\left(t \mapsto \mathbf{F}(t)+\mathbf{V}^{\text {out }}(t)\right) .\end{cases}
$$

REMARK 4.2 (Mappings defined in Definition 4.3). The above mappings are in fact the rigorous generalization of the physical process described in Section 2. To each value of the queue $\mathbf{q}$ we associate $\mathbf{h}$, the corresponding boundary flux at the entry of the intersection. From this condition, we can obtain $\overline{\mathbf{V}}_{i}^{\text {in }}$, the accumulated number of vehicles on each entry link $i \in \mathcal{I}$ and its evaluation $\mathbf{V}_{i}^{\text {in }}$ at $x=0$. Then, for each $j \in \mathcal{O}$, we can compute $\mathbf{F}_{j}$ which corresponds to the number of vehicles that reached the intersection and wish to access exit $j$. The function $\overline{\mathbf{V}}_{j}^{\text {out }}$ corresponds to the accumulated number that actually entered exit $j$ and $\mathbf{V}_{j}^{\text {out }}$ is its evaluation at $x=0$. Finally, $\boldsymbol{\Lambda}$ is the mapping that updates the queue length, by adding to the initial queue value the difference between the number of vehicles that have reached the intersection and the number of vehicles that left the junction.

Given the introduced mappings we are now in a position to formulate the proposed dynamics in Definition 3.1 of Section 3 in terms of a fixed-point problem:

Lemma 4.4 (Fixed-point problem and Definition 3.1). There exists a solution of the dynamics as proposed in Definition 3.1 if the following fixed-point problem admits a unique solution on $[0, T]$ :

$$
\mathbf{q} \equiv \mathbf{\Lambda} \circ\left(\mathbf{F} \circ \mathbf{V}^{\text {in }} \circ \mathbf{h} \circ \mathbf{q}, \mathbf{V}^{\text {out }} \circ \mathbf{F} \circ \mathbf{V}^{\text {in }} \circ \mathbf{h} \circ \mathbf{q}\right), \mathbf{q} \in C\left([0, T] ; \mathbb{R}^{|\mathcal{O}|}\right)
$$

with the involved operators as in Definition 4.3. 
So far, we have claimed in Definition 4.3 that these mappings actually map into the proper spaces. This will be justified in the next lemmas. The following trivial estimate for the maximum/minimum of Lipschitz functions is crucial:

LEмmA 4.5 (Lipschitz-continuous functions and $\max , \min$ ). Let $I \subseteq \mathbb{R}$ be given and assume $a, b \in \operatorname{Lip}(I ; \mathbb{R})$. Then, the mappings

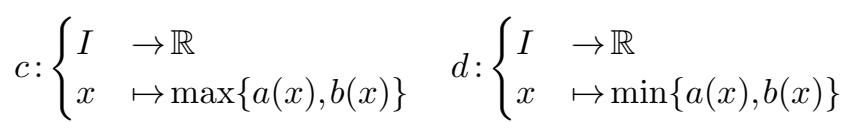

are also Lipschitz-continuous with Lipschitz-constant $\max \left\{L_{a}, L_{b}\right\}$, where $L_{a}, L_{b} \in \mathbb{R}_{\geq 0}$ denote the Lipschitz constants of the function $a, b$ respectively.

4.3. Estimates for the involved mappings. The previously introduced mappings in Definition 4.3 have to satisfy - as is assumed - Lipschitz-continuity and additional properties used later. Thus, we will present the named properties in Lemma 4.6, Proposition 4.1:

Lemma 4.6 (Space-dependent $\overline{\mathbf{V}}^{\text {out }}$ and $\left.\overline{\mathbf{V}}^{\text {in }}\right)$. Let $\mathbf{F}, \tilde{\mathbf{F}} \in \operatorname{Lip}\left([0, T] ; \mathbb{R}^{|\mathcal{O}|}\right)$ and $\mathbf{h}, \tilde{\mathbf{h}} \in$ $\operatorname{Lip}\left([0, T] ; \mathbb{R}^{|\mathcal{I}|}\right)$.

- For the space-dependent $\overline{\mathbf{V}}^{\text {out }}$ as in Definition 4.3 we obtain for $j \in \mathcal{O}$ :

$$
\begin{aligned}
& \left|\overline{\mathbf{V}}_{j}^{\text {out }}\left[\mathbf{F}_{j}\right](t, x)-\overline{\mathbf{V}}_{j}^{\text {out }}\left[\mathbf{F}_{j}\right](\tilde{t}, \tilde{x})\right| \\
& \quad \leq \boldsymbol{\rho}_{j}^{\max }|x-\tilde{x}|+\max \left\{\mathbf{f}_{j}^{\max },\left\|\mathbf{F}_{j}^{\prime}\right\|_{L^{\infty}((0, T))}\right\}|t-\tilde{t}| \quad \forall(t, x),(\tilde{t}, \tilde{x}) \in(0, T) \times \mathbb{R}_{\geq 0}, \\
& \quad\left\|\overline{\mathbf{V}}_{j}^{\text {out }}\left[\mathbf{F}_{j}\right](\cdot, 0)-\overline{\mathbf{V}}_{j}^{\text {out }}\left[\tilde{\mathbf{F}}_{j}\right](\cdot, 0)\right\|_{C([0, t])} \leq\left\|\mathbf{F}_{j}-\tilde{\mathbf{F}}_{j}\right\|_{C([0, t])} \quad \forall t \in[0, T] .
\end{aligned}
$$

- For the space-dependent $\overline{\mathbf{V}}^{\text {in }}$ as in Definition 4.3 we obtain for $i \in \mathcal{I}$ :

$$
\left\|\overline{\mathbf{V}}_{i}^{\mathrm{in}}\left[\mathbf{h}_{i}\right](\cdot, 0)-\mathbf{V}_{i}^{\mathrm{in}}\left[\tilde{\mathbf{h}}_{i}\right](\cdot, 0)\right\|_{C([0, t])} \leq\left\|\mathbf{h}_{i}-\tilde{\mathbf{h}}_{i}\right\|_{L^{1}((0, t))} \quad \forall t \in(0, T] .
$$

In addition, $\forall(t, x),(\tilde{t}, \tilde{x}) \in[0, T] \times \mathbb{R}_{<0}$ we have

$$
\begin{gathered}
\left|\overline{\mathbf{V}}_{i}^{\text {in }}\left[\mathbf{h}_{i}\right](t, x)-\overline{\mathbf{V}}_{i}^{\text {in }}\left[\mathbf{h}_{i}\right](\tilde{t}, \tilde{x})\right| \leq \boldsymbol{\rho}_{i}^{\max }|x-\tilde{x}|+\max \left\{\mathbf{f}_{i}^{\max },\left\|\mathbf{h}_{i}\right\|_{C([0, t])}\right\}|t-\tilde{t}|, \\
\frac{\mathrm{d}}{\mathrm{d} t} \overline{\mathbf{V}}_{i}^{\text {in }}\left[\mathbf{h}_{i}\right](t, 0) \leq 0 \quad \text { for a.e. } t \in(0, T), \mathbf{h}_{i} \geq 0 .
\end{gathered}
$$

Proof.

- Let $\mathbf{F} \in \operatorname{Lip}\left([0, T] ; \mathbb{R}^{|\mathcal{O}|}\right)$ and $j \in \mathcal{O}$ be given, the first term in the outer maximum of the solution formula presented for $\overline{\mathbf{V}}^{\text {out }}$ in Definition 4.3 is again Lipschitz continuous by $[19$, Section 3.3, Lemma 2] and for the second term we assume that for $(t, x) \in[0, T] \times$ $(0, L)$ the maximum is attained for $t_{1} \in[0, T]$. Then, we have for $(\tilde{t}, \tilde{x}) \in[0, T] \times[0, L]$

$$
\begin{aligned}
& \overline{\mathbf{V}}_{j}^{\text {out }}\left[\mathbf{F}_{j}\right](t, x)-\overline{\mathbf{V}}_{j}^{\text {out }}\left[\mathbf{F}_{j}\right](t, \tilde{x}) \\
& =-\mathbf{F}_{j}\left(t_{1}\right)+\left(t-t_{1}\right) \mathbf{f}_{j}^{*}\left(\frac{x}{t-t_{1}}\right)-\max _{0 \leq t_{1} \leq t}\left\{-\mathbf{F}_{j}\left(t_{1}\right)+\left(t-t_{1}\right) \mathbf{f}_{j}^{*}\left(\frac{\tilde{x}}{t-t_{1}}\right)\right\} \\
& \leq\left(t-t_{1}\right)\left(\mathbf{f}_{j}^{*}\left(\frac{x}{t-t_{1}}\right)-\mathbf{f}_{j}^{*}\left(\frac{\tilde{x}}{t-t_{1}}\right)\right) \leq \boldsymbol{\rho}_{i}^{\max }|x-\tilde{x}|
\end{aligned}
$$

by the Lipschitz-continuity of $\mathbf{f}_{j}^{*}$ as stated in Lemma 4.1. The lower bound follows analogously by exchanging $x$ and $\tilde{x}$. For the Lipschitz-continuity w.r.t. time we recall 
that for the part of the solution formula which only consists of initial datum the proof can be found - again - in [19, Section 3.3, Lemma 2] and one obtains in that case

$$
\left|\overline{\mathbf{V}}_{j}^{\text {out }}\left[\mathbf{F}_{j}\right](t, x)-\overline{\mathbf{V}}_{j}^{\text {out }}\left[\mathbf{F}_{j}\right](\tilde{t}, x)\right| \leq \mathbf{f}_{j}^{\max }|t-\tilde{t}| .
$$

For the part involving also the boundary datum assume for now that $\tilde{t} \geq t$. Then we obtain in the case that the maximum is attained at $t_{1} \in[0, t]$

$$
\begin{aligned}
& \overline{\mathbf{V}}_{j}^{\text {out }}\left[\mathbf{F}_{j}\right](t, x)-\overline{\mathbf{V}}_{j}^{\text {out }}\left[\mathbf{F}_{j}\right](\tilde{t}, x) \\
& =-\mathbf{F}_{j}\left(t_{1}\right)+\left(t-t_{1}\right) \mathbf{f}_{j}^{*}\left(\frac{x}{t-t_{1}}\right)-\max _{0 \leq \tilde{t}_{1} \leq \tilde{t}}\left\{-\mathbf{F}_{j}\left(\tilde{t}_{1}\right)+\left(\tilde{t}-\tilde{t}_{1}\right) \mathbf{f}_{j}^{*}\left(\frac{x}{\tilde{t}-\tilde{t}_{1}}\right)\right\} \\
& \tilde{t}_{1}=\tilde{t}-t+t_{1} \\
& \stackrel{\leq}{\leq}-\mathbf{F}_{j}\left(t_{1}\right)+\left(t-t_{1}\right) \mathbf{f}_{j}^{*}\left(\frac{x}{t-t_{1}}\right)+\mathbf{F}_{j}\left(\tilde{t}-t+t_{1}\right)-\left(t-t_{1}\right) \mathbf{f}_{j}^{*}\left(\frac{x}{t-t_{1}}\right) \\
& \leq\left\|\mathbf{F}_{j}^{\prime}\right\|_{L^{\infty}((0, T))}|t-\tilde{t}| .
\end{aligned}
$$

Interchanging the terms where the maximum is attained we can show the same estimate in case of $\tilde{t} \leq t$. The same argumentation can also be used to obtain a lower bound and will be omitted. Applying Lemma 4.5, we obtain the claimed estimate. For the Lipschitz-continuity w.r.t. $\mathbf{F}_{j}, \tilde{\mathbf{F}}_{j}$ we recall again that the solution formula for $\overline{\mathbf{V}}^{\text {out }}$ in Definition 4.3 only depends on $\mathbf{F}_{j}, \tilde{\mathbf{F}}_{j}$ in the second part of the maximum, so that we can only consider that part invoking Lemma 4.5. It yields then assuming that the first term takes its maximum at $t_{1} \in[0, t]$

$$
\begin{aligned}
& \overline{\mathbf{V}}_{j}^{\text {out }}\left[\mathbf{F}_{j}\right](t, 0)-\overline{\mathbf{V}}_{j}^{\text {out }}\left[\tilde{\mathbf{F}}_{j}\right](t, 0) \\
& \leq-\mathbf{F}_{j}\left(t_{1}\right)-\left(t-t_{1}\right) \mathbf{f}_{j}^{\max }-\max _{0 \leq t_{1} \leq t}\left\{-\tilde{\mathbf{F}}_{j}\left(t_{1}\right)-\left(t-t_{1}\right) \mathbf{f}_{j}^{\max }\right\} \\
& \leq \tilde{\mathbf{F}}_{j}\left(t_{1}\right)-\mathbf{F}_{j}\left(t_{1}\right) \leq\left\|\tilde{\mathbf{F}}_{j}-\mathbf{F}_{j}\right\|_{C([0, t])} .
\end{aligned}
$$

The lower bound can be obtained as usual by exchanging minuend and subtrahend in the previous estimate.

- The Lipschitz-estimate w.r.t. $\mathbf{h}, \tilde{\mathbf{h}}$ follows by standard arguments. We do not detail this.

For the Lipschitz-continuity in space and time, first note that the first part of the maximum is Lipschitz-continuous on $(t, x) \in(0, T) \times \mathbb{R}_{\leq 0}$ as a direct consequence of [19], since it involves only the initial datum.

For the second part of the maximum, things become a bit more complicated. Of course, we could refer to [32], however since the Lipschitz-constant is somewhat crucial, we will detail the proof.

Suppose $x, \tilde{x} \in \mathbb{R}_{\leq 0}$ are given. Assuming that for $(t, x) \in[0, T] \times \mathbb{R}_{\leq 0}$ the maximum in $\mathbf{V}_{i}^{\text {in }}\left[\mathbf{h}_{i}\right](t, x)$ is attained for $\left(y, t_{2}, t_{1}\right) \in \mathbb{R}_{\leq 0} \times[0, t]^{2}$ with $t_{1} \geq t_{2}$, we obtain

$$
\begin{aligned}
& \overline{\mathbf{V}}_{i}^{\mathrm{in}}\left[\mathbf{h}_{i}\right](t, x)-\overline{\mathbf{V}}_{i}^{\mathrm{in}}\left[\mathbf{h}_{i}\right](t, \tilde{x}) \\
& =\overline{\mathbf{V}}_{0, i}^{\mathrm{in}}(y)+t_{2} \mathbf{f}_{i}^{*}\left(\frac{-y}{t_{2}}\right)+\left(t-t_{1}\right) \mathbf{f}_{i}^{*}\left(\frac{x}{t-t_{1}}\right)-\int_{t_{2}}^{t_{1}} \mathbf{h}_{i}(s) \mathrm{d} s \\
& \quad-\max _{\substack{y \in \mathbb{R}_{00} \\
0 \leq t_{2} \leq t_{1} \leq \tilde{t}}}\left\{\mathbf{V}_{0, i}^{\mathrm{in}}(y)+t_{2} \mathbf{f}_{i}^{*}\left(\frac{-y}{t_{2}}\right)+\left(t-t_{1}\right) \mathbf{f}_{i}^{*}\left(\frac{\tilde{x}}{t-t_{1}}\right)-\int_{t_{2}}^{t_{1}} \mathbf{h}_{i}(s) \mathrm{d} s\right\} \\
& \leq\left(t-t_{1}\right)\left(\mathbf{f}_{i}^{*}\left(\frac{x}{t-t_{1}}\right)-\mathbf{f}_{i}^{*}\left(\frac{\tilde{x}}{t-t_{1}}\right)\right)
\end{aligned}
$$




$$
\leq\left(t-t_{1}\right) \boldsymbol{\rho}_{i}^{\max } \frac{1}{t-t_{1}}|x-\tilde{x}|=\boldsymbol{\rho}_{i}^{\max }|x-\tilde{x}|
$$

by Lemma 4.1. The analogue argumentation can be used to obtain a lower bound with the same Lipschitz-constant by assuming that the second part actually takes its maximum at a given point $\left(y, t_{2}, t_{1}\right)$ and estimating the first term from below.

For Lipschitz-continuity of the second part of the maximum w.r.t. the time variable suppose $t, \tilde{t} \in[0, T]$ be given. Then, we obtain for $t \geq \tilde{t}$ and $i \in \mathcal{I}$ by applying Lemma 4.3 in the version for concave flux function

$$
\begin{aligned}
\overline{\mathbf{V}}_{i}^{\text {in }}\left[\mathbf{h}_{i}\right](t, x)-\overline{\mathbf{V}}_{i}^{\text {in }}\left[\mathbf{h}_{i}\right](\tilde{t}, x) \\
\quad=\max _{\substack{y \in \mathbb{R}_{\leq 0} \\
\tilde{t} \leq t_{2} \leq t_{1} \leq t}}\left\{\mathbf{V}_{i}^{\text {in }}(\tilde{t}, y)+t_{2} \mathbf{f}_{i}^{*}\left(\frac{-y}{t_{2}}\right)+\left(t-t_{1}\right) \mathbf{f}_{i}^{*}\left(\frac{x}{t-t_{1}}\right)-\int_{t_{2}}^{t_{1}} \mathbf{h}_{i}(s) \mathrm{d} s-\mathbf{V}_{i}^{\text {in }}(\tilde{t}, x)\right\}
\end{aligned}
$$

using the previous estimate for the spatial Lipschitz-continuity

$$
\begin{aligned}
& \leq \max _{\substack{y \in \mathbb{R}_{\leq 00} \\
t \leq t_{2} \leq t_{1} \leq t}}\left\{\boldsymbol{\rho}_{i}^{\max }|y-x|+t_{2} \mathbf{f}_{i}^{*}\left(\frac{-y}{t_{2}}\right)+\left(t-t_{1}\right) \mathbf{f}_{i}^{*}\left(\frac{x}{t-t_{1}}\right)-\int_{t_{2}}^{t_{1}} \mathbf{h}_{i}(s) \mathrm{d} s\right\} \\
&=\max _{\substack{y \in \mathbb{R}_{\leq 0} \\
t \leq t_{2} \leq t_{1} \leq t}}\left\{\boldsymbol{\rho}_{i}^{\max }|y-x|+\left(t+t_{2}-t_{1}\right)\left(\frac{t_{2}}{t+t_{2}-t_{1}} \mathbf{f}_{i}^{*}\left(\frac{-y}{t_{2}}\right)+\frac{t-t_{1}}{t+t_{2}-t_{1}} \mathbf{f}_{i}^{*}\left(\frac{x}{t-t_{1}}\right)\right)\right. \\
&\left.-\int_{t_{2}}^{t_{1}} \mathbf{h}_{i}(s) \mathrm{d} s\right\}
\end{aligned}
$$

using the concavity of $\mathbf{f}_{i}^{*}$ as stated in Lemma 4.1

$$
\leq \max _{\substack{y \in \mathbb{R}_{\leq 0} \\ \tilde{t} \leq t_{2} \leq t_{1} \leq t}}\left\{\boldsymbol{\rho}_{i}^{\max }|y-x|+\left(t+t_{2}-t_{1}\right) \mathbf{f}_{i}^{*}\left(\frac{x-y}{t+t_{2}-t_{1}}\right)-\int_{t_{2}}^{t_{1}} \mathbf{h}_{i}(s) \mathrm{d} s\right\}
$$

substituting $z=\frac{x-y}{t+t_{2}-t_{1}}$

$$
\leq \max _{\tilde{t} \leq t_{2} \leq t_{1} \leq t}\left\{\max _{z \in\left[\frac{x}{t+t_{2}-t_{1}}, \infty\right)}\left\{\left(t+t_{2}-t_{1}\right)\left(\boldsymbol{\rho}_{i}^{\max }|z|+\mathbf{f}_{i}^{*}(z)\right)-\int_{t_{2}}^{t_{1}} \mathbf{h}_{i}(s) \mathrm{d} s\right\}\right\}
$$

setting $\tilde{z}=-z$ and using the monotonicity of $\mathbf{f}_{i}^{*}$

$$
\begin{aligned}
& \leq \max _{\tilde{t} \leq t_{2} \leq t_{1} \leq t}\left\{\max _{\tilde{z} \in\left(-\infty,-\frac{x}{t+t_{2}-t_{1}}\right]}\left\{\left(t+t_{2}-t_{1}\right)\left(\mathbf{f}_{i}^{*}(\tilde{z})-\boldsymbol{\rho}_{i}^{\max } \tilde{z}\right)-\int_{t_{2}}^{t_{1}} \mathbf{h}_{i}(s) \mathrm{d} s\right\}\right\} \\
& \leq \max _{w \in\left[0, \boldsymbol{\rho}_{i}^{\max }\right]}\left\{\max _{\substack{z \in \mathbb{R} \\
\tilde{t} \leq t_{2} \leq t_{1} \leq t}}\left\{\left(t+t_{2}-t_{1}\right)\left(\mathbf{f}_{i}^{*}(z)-w z\right)-\int_{t_{2}}^{t_{1}} \mathbf{h}_{i}(s) \mathrm{d} s\right\}\right\} \\
& =\max _{w \in\left[0, \boldsymbol{\rho}_{i}^{\max }\right]}\left\{-\min _{\substack{z \in \mathbb{R} \\
\tilde{t} \leq t_{2} \leq t_{1} \leq t}}\left\{\left(t+t_{2}-t_{1}\right)\left(w z-\mathbf{f}_{i}^{*}(z)\right)+\int_{t_{2}}^{t_{1}} \mathbf{h}_{i}(s) \mathrm{d} s\right\}\right\}
\end{aligned}
$$


using Lemma 4.1 Item 6 , stating that $\mathbf{f}^{* *} \equiv \mathbf{f}$

$$
\leq\left\|\mathbf{h}_{i}\right\|_{C([0, T]}|t-\tilde{t}| .
$$

For the lower bound, we recall that $x \in \mathbb{R}_{\leq 0}$ and estimate assuming that for $(\tilde{t}, x) \in$ $(0, t) \times \mathbb{R}_{\leq 0}$ the minimum is attained at $\left(\tilde{y}, \tilde{t}_{1}, \tilde{t}_{2}\right)$

$$
\begin{aligned}
& \overline{\mathbf{V}}_{i}^{\mathrm{in}}\left[\mathbf{h}_{i}\right](\tilde{t}, x)-\overline{\mathbf{V}}_{i}^{\mathrm{in}}\left[\mathbf{h}_{i}\right](t, x) \\
& =\mathbf{V}_{0, i}^{\mathrm{in}}(\tilde{y})+\tilde{t}_{2} \mathbf{f}_{i}^{*}\left(\frac{-\tilde{y}}{\tilde{t}_{2}}\right)+\left(\tilde{t}-\tilde{t}_{1}\right) \mathbf{f}_{i}^{*}\left(\frac{x}{\tilde{t}-\tilde{t}_{1}}\right)-\int_{\tilde{t}_{2}}^{\tilde{t}_{1}} \mathbf{h}_{i}(s) \mathrm{d} s \\
& \quad-\max _{\substack{y \in \mathbb{R}_{0} \leq 0 \\
0 \leq t_{2} \leq t_{1} \leq t}}\left\{\mathbf{V}_{0, i}^{\mathrm{in}}(y)+t_{2} \mathbf{f}_{i}^{*}\left(\frac{-y}{t_{2}}\right)+\left(t-t_{1}\right) \mathbf{f}_{i}^{*}\left(\frac{x}{t-t_{1}}\right)-\int_{t_{2}}^{t_{1}} \mathbf{h}_{i}(s) \mathrm{d} s\right\}
\end{aligned}
$$

setting $y=\tilde{y}, t_{2}=\tilde{t_{2}}, t_{1}=t-\tilde{t}+\tilde{t}_{1}$

$$
\leq \int_{\tilde{t}_{1}}^{t-\tilde{t}+\tilde{t}_{1}} \mathbf{h}_{i}(s) \mathrm{d} s \leq\left\|\mathbf{h}_{i}\right\|_{C([0, t])}|t-\tilde{t}| .
$$

Due to the previously deduced Lipschitz-estimate which guarantees the differentiability of $\overline{\mathbf{V}}^{\text {in }}$ w.r.t. space and time, it suffices to show Inequality (4.10) to prove that $t \mapsto \overline{\mathbf{V}}^{\text {in }}(t, 0)$ is monotonically decreasing. However, this has already been carried out in Eq. (4.12) as long as $\mathbf{h}_{i}$ is nonnegative (which is always the case for the considered fixed-point equation).

This concludes the proof.

Proposition 4.1 (Lipschitz-continuity of the mappings in Definition 4.3 point-wise and as operators). For the mappings in Definition 4.3 we obtain the following Lipschitzbounds when measuring in the uniform topology but also when considering them as operators. In detail:

(1) For $\mathbf{h}$ as in Eq. (4.4) with $\mathbf{q}, \tilde{\mathbf{q}} \in \operatorname{Lip}\left([0, T] ; \mathbb{R}^{|\mathcal{O}|}\right)$ we obtain for every $i \in \mathcal{I}$

$$
\begin{array}{ll}
\left|\mathbf{h}_{i}[\mathbf{q}](t)-\mathbf{h}_{i}[\mathbf{q}](\tilde{t})\right| \leq\left\|\mathbf{c}_{i}\right\|_{L^{\infty}((0, T))} \sum_{j \in \mathcal{O}}\left\|\mathbf{q}_{j}^{\prime}\right\|_{L^{\infty}((0, T))}|t-\tilde{t}| & \forall t, \tilde{t} \in[0, T], \\
\left|\mathbf{h}_{i}[\mathbf{q}](t)-\mathbf{h}_{i}[\tilde{\mathbf{q}}](t)\right| \leq\left\|\mathbf{c}_{i}\right\|_{L^{\infty}((0, t))} \sum_{j \in \mathcal{O}}\left|\mathbf{q}_{j}(t)-\tilde{\mathbf{q}}_{j}(t)\right| & \forall t \in(0, T] .
\end{array}
$$

(2) For $\mathbf{F}$ as in Eq. (4.6) with $\mathbf{V}, \tilde{\mathbf{V}} \in \operatorname{Lip}\left([0, T] ; \mathbb{R}^{|\mathcal{I}|}\right)$ we obtain for $j \in \mathcal{O}$

$$
\begin{array}{cc}
\left|\mathbf{F}_{j}[\mathbf{V}](t)-\mathbf{F}_{j}[\mathbf{V}](\tilde{t})\right| \leq \sum_{i \in \mathcal{I}}\left\|\frac{\mathrm{d}}{\mathrm{d} t} \mathbf{V}_{i}\right\|_{L^{\infty}((0, T))}|t-\tilde{t}| & \forall t, \tilde{t} \in[0, T], \\
\left\|\mathbf{F}_{j}[\mathbf{V}]-\mathbf{F}_{j}[\tilde{\mathbf{V}}]\right\|_{C([0, t])} \leq \sum_{i \in \mathcal{I}}\left(\left\|\boldsymbol{\theta}_{i, j}\right\|_{T V((0, t))}+1\right)\left\|\mathbf{V}_{i}-\tilde{\mathbf{V}}_{i}\right\|_{C([0, t])} & \forall t \in[0, T]
\end{array}
$$

(3) For $\mathbf{V}^{\text {out }}$ as in Eq. (4.7) let $\mathbf{F}, \tilde{\mathbf{F}} \in \operatorname{Lip}\left([0, T] ; \mathbb{R}^{|\mathcal{O}|}\right)$. Then, we obtain for $j \in \mathcal{O}$

$$
\begin{array}{rlrl}
\left|\mathbf{V}_{j}^{\text {out }}\left[\mathbf{F}_{j}\right](t)-\mathbf{V}_{j}^{\text {out }}\left[\mathbf{F}_{j}\right](\tilde{t})\right| & \leq\left\{\left\|\mathbf{F}_{j}^{\prime}\right\|_{L^{\infty}((0, T))}, \mathbf{f}_{j}^{\max }\right\}|t-\tilde{t}| & & \forall t, \tilde{t} \in[0, T], \\
\left\|\mathbf{V}_{j}^{\text {out }}\left[\mathbf{F}_{j}\right]-\mathbf{V}_{j}^{\text {out }}\left[\tilde{\mathbf{F}}_{j}\right]\right\|_{C([0, t])} \leq\left\|\mathbf{F}_{j}-\tilde{\mathbf{F}}_{j}\right\|_{C([0, t])} & t \in(0, T] .
\end{array}
$$


(4) For $\boldsymbol{\Lambda}$ as in Eq. (4.8) let $\left(\mathbf{F}, \mathbf{V}^{\text {out }}\right),\left(\tilde{\mathbf{F}}, \mathbf{V}^{\text {out }}\right) \in \operatorname{Lip}\left([0, T] ; \mathbb{R}^{|\mathcal{O}|}\right) \times \operatorname{Lip}\left([0, T] ; \mathbb{R}^{|\mathcal{O}|}\right)$ be given. Then, we have for $j \in \mathcal{O}$ and $\forall t, \tilde{t} \in[0, T]$

$$
\begin{gathered}
\left|\boldsymbol{\Lambda}_{j}\left[\mathbf{F}, \mathbf{V}^{\text {out }}\right](t)-\boldsymbol{\Lambda}_{j}\left[\mathbf{F}, \mathbf{V}^{\text {out }}\right](\tilde{t})\right| \leq\left(\left\|\mathbf{F}_{j}^{\prime}\right\|_{L^{\infty}((0, T))}+\left\|\frac{\mathrm{d}}{\mathrm{d} t} \mathbf{V}_{j}^{\text {out }}\right\|_{L^{\infty}((0, T))}\right)|t-\tilde{t}|, \\
\left\|\boldsymbol{\Lambda}_{j}\left[\mathbf{F}, \mathbf{V}^{\text {out }}\right]-\boldsymbol{\Lambda}_{j}\left[\tilde{\mathbf{F}}, \tilde{\mathbf{V}}^{\text {out }}\right]\right\|_{C([0, t])} \leq\left\|\mathbf{F}_{j}-\tilde{\mathbf{F}}_{j}\right\|_{C([0, t])}+\left\|\mathbf{V}_{j}^{\text {out }}-\tilde{\mathbf{V}}_{j}^{\text {out }}\right\|_{C([0, t])} .
\end{gathered}
$$

(5) For $\mathbf{V}^{\text {in }}$ as in Eq. (4.5) and $\mathbf{h}, \tilde{\mathbf{h}} \in \operatorname{Lip}\left([0, T] ; \mathbb{R}^{\mathcal{I}}\right)$ we obtain

$$
\begin{array}{rlrl}
\left|\mathbf{V}_{i}^{\text {in }}\left[\mathbf{h}_{i}\right](t)-\mathbf{V}_{i}^{\text {in }}\left[\mathbf{h}_{i}\right](\tilde{t})\right| & \leq \max \left\{\left\|\mathbf{h}_{i}\right\|_{C([0, T])}, \mathbf{f}_{i}^{\max }\right\}|t-\tilde{t}| & & \forall t, \tilde{t} \in[0, T], \\
\left\|\mathbf{V}_{i}^{\text {in }}\left[\mathbf{h}_{i}\right]-\mathbf{V}_{i}^{\text {in }}\left[\tilde{\mathbf{h}}_{i}\right]\right\|_{C([0, t])} \leq\left\|\mathbf{h}_{i}-\tilde{\mathbf{h}}_{i}\right\|_{L^{1}((0, t))} & & \forall t \in(0, T] .
\end{array}
$$

Proof.

(1) We apply Lemma 4.5 and have, due to the Lipschitz-constant of $\mathbf{f}_{i}$ being zero,

$$
\left|\mathbf{h}_{i}[\mathbf{q}](t)-\mathbf{h}_{i}[\mathbf{q}](\tilde{t})\right| \leq\left\|\mathbf{c}_{i}\right\|_{L^{\infty}((0, T))} \sum_{j \in \mathcal{O}}\left\|\mathbf{q}_{j}^{\prime}\right\|_{L^{\infty}((0, T))}|t-\tilde{t}|
$$

For the second inequality, we assume first for $t \in[0, T]$ given that $\mathbf{f}_{i}^{\max } \geq \mathbf{c}_{i}(t)(M-$ $\left.\sum_{j \in \mathcal{O}} \tilde{\mathbf{q}}_{j}(t)\right)$. Then, we obtain

$$
\begin{aligned}
\mathbf{h}_{i}[\mathbf{q}](t)-\mathbf{h}_{i}[\tilde{\mathbf{q}}](t) & =\min \left\{\mathbf{f}_{i}^{\max }, \mathbf{c}_{i}(t)\left(M-\sum_{j \in \mathcal{O}} \mathbf{q}_{j}(t)\right)\right\}-\mathbf{c}_{i}(t)\left(M-\sum_{j \in \mathcal{O}} \tilde{\mathbf{q}}_{j}(t)\right) \\
& \leq\left|\mathbf{c}_{i}(t)\right| \sum_{j \in \mathcal{O}}\left|\mathbf{q}_{j}(t)-\tilde{\mathbf{q}}_{j}(t)\right| .
\end{aligned}
$$

For $\mathbf{f}_{i}^{\max } \leq \mathbf{c}_{i}(t)\left(M-\sum_{j \in \mathcal{O}} \tilde{\mathbf{q}}_{j}(t)\right)$ we obtain

$$
\begin{aligned}
& \mathbf{h}_{i}[\mathbf{q}](t)-\mathbf{h}_{i}[\tilde{\mathbf{q}}](t) \\
& \leq \min \left\{\mathbf{f}_{i}^{\max }, \mathbf{c}_{i}(t)\left(M-\sum_{j \in \mathcal{O}} \mathbf{q}_{j}(t)\right)\right\}-\mathbf{f}_{i}^{\max } \leq 0 \leq\left|\mathbf{c}_{i}(t)\right| \sum_{j \in \mathcal{O}}\left|\mathbf{q}_{j}(t)-\tilde{\mathbf{q}}_{j}(t)\right| .
\end{aligned}
$$

The lower bound can be obtained by exchanging $\mathbf{h}_{i}[\mathbf{q}]$ with $\mathbf{h}_{i}[\tilde{\mathbf{q}}]$ and applying the same reasoning.

(2) We estimate directly the formula for $\mathbf{F}$ and recall the uniform bound on $\boldsymbol{\theta}$ in Eq. (3.1)

$$
\begin{aligned}
\left|\mathbf{F}_{j}[\mathbf{V}](t)-\mathbf{F}_{j}[\mathbf{V}](\tilde{t})\right| & =\left|\sum_{i \in \mathcal{I}} \int_{0}^{t} \boldsymbol{\theta}_{i, j}(s) \frac{\mathrm{d}}{\mathrm{d} s} \mathbf{V}_{i}(s) \mathrm{d} s-\int_{0}^{\tilde{t}} \boldsymbol{\theta}_{i, j}(s) \frac{\mathrm{d}}{\mathrm{d} s} \mathbf{V}_{i}(s) \mathrm{d} s\right| \\
& \leq \sum_{i \in \mathcal{I}}\left|\int_{t}^{\tilde{t}} \boldsymbol{\theta}_{i, j}(s) \frac{\mathrm{d}}{\mathrm{d} s} \mathbf{V}_{i}(s) \mathrm{d} s\right| \leq \sum_{i \in \mathcal{I}}|t-\tilde{t}|\left\|\frac{\mathrm{d}}{\mathrm{d} s} \mathbf{V}_{i}\right\|_{C([0, \max t, \tilde{t}])}
\end{aligned}
$$


For the second estimate we require an integration by parts formula for functions of $B V$ functions so that we have

$$
\begin{aligned}
\left|\mathbf{F}_{j}[\mathbf{V}](t)-\mathbf{F}_{j}[\tilde{\mathbf{V}}](t)\right| & =\left|\sum_{i \in \mathcal{I}} \int_{0}^{t} \boldsymbol{\theta}_{i, j}(s) \frac{\mathrm{d}}{\mathrm{d} s}\left(\mathbf{V}_{i}(s)-\tilde{\mathbf{V}}_{i}(s)\right) \mathrm{d} s\right| \\
& =\sum_{i \in \mathcal{I}} \mid-\int_{0}^{t}\left(\mathbf{V}_{i}(s)-\tilde{\mathbf{V}}_{i}(s)\right) \mathrm{d} \boldsymbol{\theta}_{i, j}^{\prime}(s)+\boldsymbol{\theta}_{i, j}(t)\left(\mathbf{V}_{i}(t)-\tilde{\mathbf{V}}_{i}(t)\right) \\
& -\boldsymbol{\theta}_{i, j}(0)\left(\mathbf{V}_{i}(0)-\tilde{\mathbf{V}}_{i}(0)\right) \mid \\
& \leq \sum_{i \in \mathcal{I}}\left(\left\|\mathbf{V}_{i}-\tilde{\mathbf{V}}_{i}\right\|_{C([0, t])}\left\|\boldsymbol{\theta}_{i, j}\right\|_{T V((0, t))}+\left\|\mathbf{V}_{i}-\tilde{\mathbf{V}}_{i}\right\|_{C([0, t])}\right) .
\end{aligned}
$$

(3) See Lemma 4.6 as this directly follows when reconsidering $\overline{\mathbf{V}}^{\text {out }}(\cdot, 0) \equiv \mathbf{V}^{\text {out }}$ as stated in Definition 4.3.

(4) This follows directly by the linearity of the operator $\boldsymbol{\Lambda}$.

(5) See Lemma 4.6 as this directly follows when reconsidering $\overline{\mathbf{V}}^{\text {in }}(\cdot, 0) \equiv \mathbf{V}^{\text {in }}$ as stated in Definition 4.3.

Corollary 4.1 (Well-posedness of the fixed-point mapping Lemma 4.4). The mapping

$$
\mathfrak{G}:=\mathbf{\Lambda} \circ\left(\mathbf{F} \circ \mathbf{V}^{\text {in }} \circ \mathbf{h} \circ \cdot \mathbf{V}^{\text {out }} \circ \mathbf{F} \circ \mathbf{V}^{\text {in }} \circ \mathbf{h} \circ \cdot\right): \operatorname{Lip}\left([0, T] ; \mathbb{R}^{|\mathcal{O}|}\right) \rightarrow \operatorname{Lip}\left([0, T] ; \mathbb{R}^{|\mathcal{O}|}\right)
$$

explicitly given in Definition 4.3 is well-defined.

Proof. Using the aforementioned Proposition 4.1, particularly Inequalities (4.13), (4.15), (4.17), (4.19) and (4.21), this is a direct consequence.

4.4. Existence and Uniqueness. Being prepared with the estimates on the involved functions in Section 4.3 we now attack the fixed-point problem describing the dynamics at the intersections and prove existence and uniqueness of solutions in the proper topological setup.

ThEOREM 4.3 (Existence and uniqueness of a fixed-point). Let $T \in \mathbb{R}_{>0}$ be given, the fixed-point problem

$$
\mathfrak{G}[\mathbf{q}]=\mathbf{q} \quad \text { in } \boldsymbol{\Omega}_{T}
$$

as defined in Lemma 4.4 has a unique solution with

$$
\boldsymbol{\Omega}_{T}:=\left\{\mathbf{q} \in \operatorname{Lip}\left([0, T] ; \mathbb{R}_{\geq 0}^{|\mathcal{O}|}\right):\left\|\mathbf{q}_{j}^{\prime}\right\|_{L^{\infty}((0, T))} \leq 2 \max \left\{\sum_{i \in \mathcal{I}} \mathbf{f}_{i}^{\max }, \mathbf{f}_{j}^{\max }\right\} \forall j \in \mathcal{O}\right\}
$$

Proof. As mentioned before, we only prove the Single-Buffer/Multi-Queues case in Item 2, Definition 3.1. Pick $T^{*} \in[0, T]$ and $\mathbf{q} \in \boldsymbol{\Omega}_{T^{*}}$. Then, we aim for showing first that $\mathfrak{G}\left[\boldsymbol{\Omega}_{T^{*}}\right] \subseteq \boldsymbol{\Omega}_{T^{*}}$, i.e. $\mathfrak{G}[\mathbf{q}]$ is a self-mapping. By the previous well-posedness of the fixed-point mapping in Corollary 4.1 it suffices to show that the weak derivative of $\mathfrak{G}$ satisfies the postulated bounds. Recalling the definition of $\mathfrak{G}$ in Lemma 4.4 we thus obtain for $j \in \mathcal{O}$ and $t \in\left[0, T^{*}\right]$ a.e.

$$
\left|\frac{\mathrm{d}}{\mathrm{d} t} \mathfrak{G}_{j}[\mathbf{q}](t)\right|=\left|\frac{\mathrm{d}}{\mathrm{d} t} \boldsymbol{\Lambda}_{j}\left[\mathbf{F} \circ \mathbf{V}^{\text {in }} \circ \mathbf{h} \circ \mathbf{q}, \mathbf{V}^{\text {out }} \circ \mathbf{F} \circ \mathbf{V}^{\text {in }} \circ \mathbf{h} \circ \mathbf{q}\right](t)\right|
$$


applying Inequality (4.19)

$$
\leq\left(\left\|\frac{\mathrm{d}}{\mathrm{d} t} \mathbf{F}_{j}\left[\mathbf{V}^{\text {in }} \circ \mathbf{h} \circ \mathbf{q}\right]\right\|_{L^{\infty}((0, T))}+\left\|\frac{\mathrm{d}}{\mathrm{d} t} \mathbf{V}_{j}^{\text {out }}\left[\mathbf{F} \circ \mathbf{V}^{\text {in }} \circ \mathbf{h} \circ \mathbf{q}\right]\right\|_{L^{\infty}((0, T))}\right)
$$

applying Inequality (4.17)

$$
\leq 2 \max \left\{\left\|\frac{\mathrm{d}}{\mathrm{d} t} \mathbf{F}_{j}\left[\mathbf{V}^{\mathrm{in}} \circ \mathbf{h} \circ \mathbf{q}\right]\right\|_{L^{\infty}((0, T))}, \mathbf{f}_{j}^{\max }\right\}
$$

applying Inequality (4.15)

$$
\leq 2 \max \left\{\sum_{i \in \mathcal{I}}\left\|\frac{\mathrm{d}}{\mathrm{d} t} \mathbf{V}_{i}^{\mathrm{in}}[\mathbf{h} \circ \mathbf{q}]\right\|_{L^{\infty}((0, T))}, \mathbf{f}_{j}^{\max }\right\}
$$

applying Inequality (4.21) and the uniform bound on $\boldsymbol{\theta}$ as in Eq. (3.1)

$$
\leq 2 \max \left\{\sum_{i \in \mathcal{I}} \max \left\{\left\|\mathbf{h}_{i}[\mathbf{q}]\right\|_{L^{\infty}((0, T))}, \mathbf{f}_{i}^{\max }\right\}, \mathbf{f}_{j}^{\max }\right\}
$$

applying the definition of $\mathbf{h}$ in Eq. (4.4) Definition 4.3

$$
\begin{aligned}
& \leq 2 \max \left\{\sum _ { i \in \mathcal { I } } \operatorname { m a x } \left\{\mathbf{f}_{i}^{\max }, \min \left\{\mathbf{f}_{i}^{\max },\right.\right.\right. \\
& \left.\left.\left.\left\|\mathbf{c}_{i}\right\|_{L^{\infty}((0, T))}\left\|M-\sum_{j \in J} \mathbf{q}_{j}\right\|_{C([0, T])}\right\}\right\}, \mathbf{f}_{j}^{\max }\right\} \\
& \leq 2 \max \left\{\sum_{i \in \mathcal{I}} \mathbf{f}_{i}^{\max }, \mathbf{f}_{j}^{\max }\right\} .
\end{aligned}
$$

This is the claimed estimate. For the positivity, we pick again $j \in \mathcal{O}$ and $t \in[0, T]$ and estimate for $\mathbf{q} \in \boldsymbol{\Omega}_{T^{*}}$ applying Definition 4.3

$$
\begin{aligned}
\mathfrak{G}_{j}[\mathbf{q}](t) & =\boldsymbol{\Lambda}_{j}\left[\mathbf{F} \circ \mathbf{V}^{\text {in }} \circ \mathbf{h} \circ \mathbf{q}, \mathbf{V}^{\text {out }} \circ \mathbf{F} \circ \mathbf{V}^{\text {in }} \circ \mathbf{h} \circ \mathbf{q}\right](t) \\
& =\mathbf{F}_{j}\left[\mathbf{V}^{\text {in }} \circ \mathbf{h} \circ \mathbf{q}\right](t)+\mathbf{V}_{j}^{\text {out }}\left[\mathbf{F} \circ \mathbf{V}^{\text {in }} \circ \mathbf{h} \circ \mathbf{q}\right](t)
\end{aligned}
$$

applying Definition 4.3

$$
\geq \mathbf{F}_{j}\left[\mathbf{V}^{\text {in }} \circ \mathbf{h} \circ \mathbf{q}\right](t)+\max _{0 \leq t_{1} \leq t}\left\{-\mathbf{F}_{j}\left[\mathbf{V}^{\text {in }} \circ \mathbf{h} \circ \mathbf{q}\right]\left(t_{1}\right)-\left(t-t_{1}\right) \mathbf{f}_{j}^{\max }\right\} \geq 0
$$

by choosing $t_{1}=t$; which is independent of $\mathbf{q}$. This is true for any time $t \in[0, T]$ and every $j \in \mathcal{O}$ so that we obtain the lower bound.

As a next step we will show that the fixed-point mapping $\mathfrak{G}$ is a contraction in $\boldsymbol{\Omega}_{t}$ as defined in Eq. (4.23) for sufficiently small time horizon in the uniform topology. Thus, let $\mathbf{q}, \tilde{\mathbf{q}} \in \boldsymbol{\Omega}_{T^{*}}$ be given as well as $t \in[0, T]$. Then, we obtain by applying the fixed-point equation in Lemma 4.4 for $j \in \mathcal{O}$

$$
\begin{aligned}
& \left|\mathfrak{G}_{j}[\mathbf{q}](t)-\mathfrak{G}_{j}[\tilde{\mathbf{q}}](t)\right| \\
& \leq \mid \boldsymbol{\Lambda}_{j}\left[\mathbf{F} \circ \mathbf{V}^{\text {in }} \circ \mathbf{h} \circ \mathbf{q}, \mathbf{V}^{\text {out }} \circ \mathbf{F} \circ \mathbf{V}^{\text {in }} \circ \mathbf{h} \circ \mathbf{q}\right](t)
\end{aligned}
$$




$$
-\boldsymbol{\Lambda}_{j}\left[\mathbf{F} \circ \mathbf{V}^{\text {in }} \circ \mathbf{h} \circ \tilde{\mathbf{q}}, \mathbf{V}^{\text {out }} \circ \mathbf{F}_{j} \circ \mathbf{V}^{\text {in }} \circ \mathbf{h} \circ \tilde{\mathbf{q}}\right](t)
$$

applying Definition 4.3 and Inequality (4.20)

$$
\begin{aligned}
\leq \mid \mathbf{F}_{j} & {\left[\mathbf{V}^{\text {in }} \circ \mathbf{h} \circ \mathbf{q}\right](t)-\mathbf{F}_{j}\left[\mathbf{V}^{\text {in }} \circ \mathbf{h} \circ \tilde{\mathbf{q}}\right](t) \mid } \\
+ & \left|\mathbf{V}_{j}^{\text {out }}\left[\mathbf{F} \circ \mathbf{V}^{\text {in }} \circ \mathbf{h} \circ \mathbf{q}\right](t)-\mathbf{V}_{j}^{\text {out }}\left[\mathbf{F} \circ \mathbf{V}^{\text {in }} \circ \mathbf{h} \circ \tilde{\mathbf{q}}\right](t)\right|
\end{aligned}
$$

applying Definition 4.3 and Inequality (4.18)

$$
\begin{aligned}
& \leq\left|\mathbf{F}_{j}\left[\mathbf{V}^{\text {in }} \circ \mathbf{h} \circ \mathbf{q}\right](t)-\mathbf{F}_{j}\left[\mathbf{V}^{\text {in }} \circ \mathbf{h} \circ \tilde{\mathbf{q}}\right](t)\right|+\left\|\mathbf{F}_{j}\left[\mathbf{V}^{\text {in }} \circ \mathbf{h} \circ \mathbf{q}\right]-\mathbf{F}_{j}\left[\mathbf{V}^{\text {in }} \circ \mathbf{h} \circ \tilde{\mathbf{q}}\right]\right\|_{C([0, t])} \\
& \leq 2\left\|\mathbf{F}_{j}\left[\mathbf{V}^{\text {in }} \circ \mathbf{h} \circ \mathbf{q}\right]-\mathbf{F}_{j}\left[\mathbf{V}^{\text {in }} \circ \mathbf{h} \circ \tilde{\mathbf{q}}\right]\right\|_{C([0, t])}
\end{aligned}
$$

applying Definition 4.3 and Inequality (4.16)

$$
\leq 2 \sum_{i \in \mathcal{I}}\left(\left|\boldsymbol{\theta}_{i, j}\right|_{T V((0, t))}+1\right)\left\|\mathbf{V}_{i}^{\text {in }}[\mathbf{h} \circ \mathbf{q}]-\mathbf{V}_{i}^{\text {in }}[\mathbf{h} \circ \tilde{\mathbf{q}}]\right\|_{C([0, t])}
$$

applying Definition 4.3 and Inequality (4.22)

$$
\leq 2 \sum_{i \in \mathcal{I}}\left(\left|\boldsymbol{\theta}_{i, j}\right|_{T V((0, t))}+1\right)\left\|\mathbf{h}_{i}[\mathbf{q}]-\mathbf{h}_{i}[\tilde{\mathbf{q}}]\right\|_{L^{1}((0, t))}
$$

applying Definition 4.3 and Inequality (4.14) as well as Hölder's inequality to estimate the $L^{1}$ norm

$$
\leq 2 t \sum_{i \in \mathcal{I}}\left(\left|\boldsymbol{\theta}_{i, j}\right|_{T V((0, t))}+1\right)\left\|\mathbf{c}_{i}\right\|_{L^{\infty}((0, T))} \sum_{j \in \mathcal{O}}\left\|\mathbf{q}_{j}-\tilde{\mathbf{q}}_{j}\right\|_{C([0, t])} .
$$

Since the presented estimate is uniform in $t$, we can sum over $j \in \mathcal{O}$ and obtain

$$
\begin{aligned}
& \sum_{j \in \mathcal{O}}\left\|\mathfrak{G}_{j}[\mathbf{q}]-\mathfrak{G}_{j}[\tilde{\mathbf{q}}]\right\|_{C([0, t])} \\
& \leq 2 t\|\mathbf{c}\|_{L^{\infty}\left((0, T) ; \mathbb{R}^{|\mathcal{I}|}\right)}\left(\sum_{(i, j) \in \mathcal{I} \times \mathcal{O}}\left(\left|\boldsymbol{\theta}_{i, j}\right|_{T V((0, T))}+1\right)\right) \sum_{j \in \mathcal{O}}\left\|\mathbf{q}_{j}-\tilde{\mathbf{q}}_{j}\right\|_{C([0, t])} .
\end{aligned}
$$

Thus, picking $T^{*} \in(0, T]$

$$
T^{*}:=\frac{1}{4\|\mathbf{c}\|_{L^{\infty}((0, T) ; \mathbb{R}|\mathcal{I}|)}\left(\sum_{(i, j) \in \mathcal{I} \times \mathcal{O}}\left(\left|\boldsymbol{\theta}_{i, j}\right|_{T V((0, T))}+1\right)\right)}>0
$$

it yields

$$
\sum_{j \in \mathcal{O}}\left\|\mathfrak{G}_{j}[\mathbf{q}]-\mathfrak{G}_{j}[\tilde{\mathbf{q}}]\right\|_{C\left(\left[0, T^{*}\right]\right)} \leq \frac{1}{2} \sum_{j \in \mathcal{O}}\left\|\mathbf{q}_{j}-\tilde{\mathbf{q}}_{j}\right\|_{C\left(\left[0, T^{*}\right]\right)}
$$

so that $\mathfrak{G}$ is a self-mapping and a contraction on $\boldsymbol{\Omega}_{T^{*}}$. Since $\boldsymbol{\Omega}_{T^{*}}$ is closed in the uniform topology, we can apply Banach's fixed-point theorem [39, Theorem 1.A] and obtain a unique solution of the fixed-point problem stated in Lemma 4.4.

The assumption in $T^{*}$ being sufficiently small is not restrictive. Due to the semigroup property of the dynamical system one can "reinitialize" the fixed-point problem at time $t=T^{*}$ with new initial queue load, and initial and boundary datum on in- 
and out-going edges. However, as neither the self-mapping property nor the contraction depends on those data, we can apply the same reasoning as above to extend the solution to the time horizon $\left[0,2 T^{*}\right]$. This can be iterated until one arrives at the final time $T$.

Lemma 4.7 (q respecting the size $M$ of the buffer). The unique fixed-point $\mathbf{q}^{*} \in \boldsymbol{\Omega}_{T}$ of the fixed-point mappings stated in Lemma 4.4 and Theorem 4.3 satisfies for every $t \in[0, T]$

$$
0 \leq \sum_{j \in \mathcal{O}} \mathbf{q}_{j}^{*}(t) \leq M
$$

Proof. The lower bound has already been shown in the proof of Theorem 4.3.

Next, we will provide the upper bound. For that, we use an argument based on Gronwall's Lemma and perform it for $j \in \mathcal{O}$ and $t \in[0, T]$ by recalling that $\mathbf{q}^{*}$ is the unique solution of the fixed-point equation in Lemma 4.4, guaranteed by Theorem 4.3

$$
\mathbf{q}_{j}^{*}(t)=\mathfrak{G}_{j}\left[\mathbf{q}^{*}\right](t)
$$

applying Lemma 4.4

$$
=\mathbf{F}_{j}\left[\mathbf{V}^{\text {in }} \circ \mathbf{h} \circ \mathbf{q}^{*}\right](t)+\mathbf{V}_{j}^{\text {out }}\left[\mathbf{F}_{j} \circ \mathbf{V}^{\text {in }} \circ \mathbf{h} \circ \mathbf{q}^{*}\right](t)
$$

applying Definition 4.3

$$
=\mathbf{F}_{j}\left[\mathbf{V}^{\mathrm{in}} \circ \mathbf{h} \circ \mathbf{q}^{*}\right](t)+\max _{t_{1} \in[0, t]}\left\{-\mathbf{F}_{j}\left[\mathbf{V}^{\text {in }} \circ \mathbf{h} \circ \mathbf{q}^{*}\right]\left(t_{1}\right)-\left(t-t_{1}\right) \mathbf{f}_{j}^{\max }\right\}
$$

applying that $\mathbf{F}_{j} \geq 0$ due to Definition 4.3 and Inequality (4.10)

$$
\leq \mathbf{F}_{j}\left[\mathbf{V}^{\text {in }} \circ \mathbf{h} \circ \mathbf{q}^{*}\right](t)
$$

applying Definition 4.3

$$
\leq \mathbf{q}_{0, j}-\sum_{i \in \mathcal{I}} \int_{0}^{t} \frac{\mathrm{d}}{\mathrm{d} s} \mathbf{V}_{i}^{\mathrm{in}}\left[\mathbf{h} \circ \mathbf{q}^{*}\right](s) \boldsymbol{\theta}_{i, j}(s) \mathrm{d} s .
$$

Summarizing w.r.t. $j \in \mathcal{O}$ we obtain then

$$
\sum_{j \in \mathcal{O}} \mathbf{q}_{j}^{*}(t) \leq \sum_{j \in \mathcal{O}} \mathbf{q}_{0, j}-\sum_{i \in \mathcal{I}} \int_{0}^{t} \frac{\mathrm{d}}{\mathrm{d} s} \mathbf{V}_{i}^{\mathrm{in}}\left[\mathbf{h} \circ \mathbf{q}^{*}\right](s) \sum_{j \in \mathcal{O}} \boldsymbol{\theta}_{i, j}(s) \mathrm{d} s
$$

using Eq. (3.1)

$$
\begin{aligned}
& =\sum_{j \in \mathcal{O}} \mathbf{q}_{0, j}-\sum_{i \in \mathcal{I}} \int_{0}^{t} \frac{\mathrm{d}}{\mathrm{d} s} \mathbf{V}_{i}^{\mathrm{in}}\left[\mathbf{h} \circ \mathbf{q}^{*}\right](s) \mathrm{d} s \\
& =\sum_{j \in \mathcal{O}} \mathbf{q}_{0, j}-\sum_{i \in \mathcal{I}} \mathbf{V}_{i}^{\mathrm{in}}\left[\mathbf{h} \circ \mathbf{q}^{*}\right](t)+\sum_{i \in \mathcal{I}} \mathbf{V}_{i}^{\mathrm{in}}\left[\mathbf{h} \circ \mathbf{q}^{*}\right](0)
\end{aligned}
$$

using Definition 4.2 and Definition 4.3

$$
=\sum_{j \in \mathcal{O}} \mathbf{q}_{0, j}+\sum_{i \in \mathcal{I}} \mathbf{V}_{i}^{\mathrm{in}}(0)
$$




$$
\begin{aligned}
&-\sum_{i \in \mathcal{I}} \max _{\substack{y \in \mathbb{R}_{\leq 0} \\
0 \leq t_{2} \leq t_{1} \leq t}}\left\{\mathbf{V}_{i}^{\mathrm{in}}(y)+t_{2} \mathbf{f}_{i}^{*}\left(\frac{y}{t_{2}}\right)-\left(t-t_{1}\right) \mathbf{f}_{i}^{\mathrm{max}}-\int_{t_{2}}^{t_{1}}(\mathbf{h} \circ \mathbf{q})_{i}(s) \mathrm{d} s\right\} \\
& \leq \sum_{j \in \mathcal{O}} \mathbf{q}_{0, j}+\sum_{i \in \mathcal{I}} \mathbf{V}_{i}^{\mathrm{in}}(0) \\
& \quad-\sum_{i \in \mathcal{I}} \max _{0 \leq t_{2} \leq t_{1} \leq t}\left\{\mathbf{V}_{i}^{\mathrm{in}}(0)-\left(t+t_{2}-t_{1}\right) \mathbf{f}_{i}^{\max }-\int_{t_{2}}^{t_{1}}(\mathbf{h} \circ \mathbf{q})_{i}(s) \mathrm{d} s\right\}
\end{aligned}
$$

choosing $t_{2}=0$ and $t_{1}=t$

$$
\leq \sum_{j \in \mathcal{O}} \mathbf{q}_{0, j}+\sum_{i \in \mathcal{I}} \int_{0}^{t} \mathbf{h}_{i}\left[\mathbf{q}^{*}\right](s) \mathrm{d} s
$$

using Definition 4.3

$$
\begin{aligned}
& \leq \sum_{j \in \mathcal{O}} \mathbf{q}_{0, j}+\sum_{i \in \mathcal{I}} \int_{0}^{t} \min \left\{\mathbf{f}_{i}^{\max }, \mathbf{c}_{i}(s)\left(M-\sum_{j \in \mathcal{O}} \mathbf{q}_{j}^{*}(s)\right)\right\} \mathrm{d} s \\
& \leq \sum_{j \in \mathcal{O}} \mathbf{q}_{0, j}+\sum_{i \in \mathcal{I}}\left\|\mathbf{c}_{i}\right\|_{L^{\infty}((0, t))}\left(M t-\int_{0}^{t} \sum_{j \in \mathcal{O}} \mathbf{q}_{j}^{*}(s) \mathrm{d} s\right) .
\end{aligned}
$$

Assuming that there exists $t_{0}$ such that $\sum_{j \in \mathcal{O}} \mathbf{q}_{j}^{*}\left(t_{0}\right)>M$. Then, due to the continuity of $\mathbf{q}_{j}^{*}$ there exists a $t_{*} \in\left[0, t_{0}\right)$ such that $\sum_{j \in \mathcal{O}} \mathbf{q}_{j}^{*}\left(t_{*}\right)=M$ and $\sum_{j \in \mathcal{O}} \mathbf{q}_{j}^{*}(t)>$ $M \forall t \in\left(t_{*}, t_{0}\right)$. Integrating $\sum_{j \in \mathcal{O}} \mathbf{q}_{j}^{*}(\cdot)$ between $t_{*}$ and $t_{0}$ yields

$$
\int_{t_{*}}^{t} \sum_{j \in \mathcal{O}} \mathbf{q}_{j}^{*}(s) \mathrm{d} s>M\left(t-t_{*}\right) \quad \forall t \in\left(t_{*}, t_{0}\right) .
$$

Using the previously deduced integral inequality, we obtain for $t \in\left[t_{*}, t_{0}\right]$

$$
\begin{aligned}
\sum_{j \in \mathcal{O}} \mathbf{q}_{j}^{*}(t) & \leq \sum_{j \in \mathcal{O}} \mathbf{q}_{j}^{*}\left(t_{*}\right)+\sum_{i \in \mathcal{I}}\left\|\mathbf{c}_{i}\right\|_{L^{\infty}((0, T))} \int_{t_{*}}^{t} M-\sum_{j \in \mathcal{O}} \mathbf{q}_{j}^{*}(s) \mathrm{d} s \\
& \leq M+\sum_{i \in \mathcal{I}}\left\|\mathbf{c}_{i}\right\|_{L^{\infty}((0, T))} \int_{t_{*}}^{t} \sum_{j \in \mathcal{O}} \mathbf{q}_{j}^{*}(s)-M \mathrm{~d} s
\end{aligned}
$$

which is equivalent to

$$
\sum_{j \in \mathcal{O}} \mathbf{q}_{j}^{*}(t)-M \leq \sum_{i \in \mathcal{I}}\left\|\mathbf{c}_{i}\right\|_{L^{\infty}((0, T))} \int_{t_{*}}^{t} \sum_{j \in \mathcal{O}} \mathbf{q}_{j}^{*}(s)-M \mathrm{~d} s, \quad t \in\left[t_{*}, t_{0}\right] .
$$

Then by Grönwall's inequality, we obtain

$$
\sum_{j \in \mathcal{O}} \mathbf{q}_{j}^{*}(t)-M \leq 0 \Longrightarrow \sum_{j \in \mathcal{O}} \mathbf{q}_{j}^{*}(t) \leq M \quad t \in\left[t_{*}, t_{0}\right] .
$$

This contradicts the assumption that $\exists t_{0} \in[0, T] \sum_{j \in \mathcal{O}} \mathbf{q}_{j}^{*}\left(t_{0}\right)>M$ so that we can conclude that $\sum_{j \in \mathcal{O}} \mathbf{q}_{j}^{*}(t) \leq M \forall t \in[0, T]$.

REMARK 4.3 (Lack of uniform BV bounds on the solution). Note that we have not studied whether the corresponding solutions at the level of conservation laws admit uniform $B V$ bounds as we were approaching the problem by means of Hamilton-Jacobi equations. As outlined in [2], uniform BV estimates do not hold in general. Further analysis in this sense is out of the scope of this contribution. 


\section{Stability w.r.t. initial datum, initial queue size and routing}

In this section, we study how the introduced junction model behaves under perturbations. We aim for the most sharp stability result while still obtaining a uniform convergence of the solution. Due to the importance of the queue, we show its uniform convergence when measuring the input datum in $L^{1}$ which then enables us to obtain also the stability of the solutions of the PDEs on the incoming and outgoing links.

LEMMA 5.1 (Lipschitz-continuity w.r.t. initial datum and routing). We provide the following stability estimates for the mappings defined in Definition 4.3 with respect to initial datum, initial queue size, and routing ratio.

(1) Let $\mathbf{h} \in \operatorname{Lip}\left([0, T] ; \mathbb{R}^{|\mathcal{I}|}\right)$ be given. Recall Definition 4.3 and let $\mathbf{V}^{\text {in }}[\mathbf{h}]$ and $\tilde{\mathbf{V}}^{\text {in }}[\mathbf{h}]$ respectively correspond to the solution obtained when computing $\mathbf{V}^{\text {in }}[\mathbf{h}]$ from $\boldsymbol{\rho}_{0, i} \in$ $L^{1}((-\infty, 0))$ or $\tilde{\boldsymbol{\rho}}_{0, i} \in L^{1}((-\infty, 0))$ for any $i \in \mathcal{I}$ with initial datum of the conservation law associated with the initial datum of the Hamilton-Jacobi equation as in Definition 4.2. Then, we have the following estimate:

$$
\left\|\mathbf{V}_{i}^{\text {in }}[\mathbf{h}]-\tilde{\mathbf{V}}_{i}^{\text {in }}[\mathbf{h}]\right\|_{C([0, T])} \leq\left\|\boldsymbol{\rho}_{0, i}-\tilde{\boldsymbol{\rho}}_{0, i}\right\|_{L^{1}((-\infty, 0))} .
$$

(2) $\operatorname{Let} \mathbf{V} \in \operatorname{Lip}\left([0, T] ; \mathbb{R}^{|\mathcal{I}|}\right)$ with $\left\|\mathbf{V}_{i}^{\prime}\right\|_{L^{\infty}((0, T))} \leq \mathbf{f}_{i}^{\max }$ for $i \in \mathcal{I}$ and $\mathbf{F}[\mathbf{V}]$ and $\tilde{\mathbf{F}}[\mathbf{V}]$ respectively correspond to the value obtained when computing $\mathbf{F}$ from $\mathbf{q}_{0}, \boldsymbol{\theta}$ and $\tilde{\mathbf{q}}_{0}, \tilde{\boldsymbol{\theta}}$ with $\boldsymbol{\theta}, \tilde{\boldsymbol{\theta}} \in L^{1}\left(\left((0, T) ; \mathbb{R}^{|\mathcal{O}||\mathcal{I}|}\right)\right)$. Then, we have the following estimate:

$$
\forall j \in \mathcal{O}:\left\|\mathbf{F}_{j}[\mathbf{V}]-\tilde{\mathbf{F}}_{j}[\mathbf{V}]\right\|_{C([0, T])} \leq\left|\mathbf{q}_{0, j}-\tilde{\mathbf{q}}_{0, j}\right|+\sum_{i \in \mathcal{I}} \mathbf{f}_{\max }^{i}\left\|\boldsymbol{\theta}_{i, j}-\tilde{\boldsymbol{\theta}}_{i, j}\right\|_{L^{1}((0, T))}
$$

(3) Let $\mathbf{F} \in \operatorname{Lip}\left([0, T] ; \mathbb{R}^{|\mathcal{O}|}\right)$ and $\mathbf{V}^{\text {out }}[\mathbf{F}]$ and $\tilde{\mathbf{V}}^{\text {out }}[\mathbf{F}]$ respectively correspond to the value obtained when computing $\mathbf{V}^{\text {out }}[\mathbf{F}]$ from $\boldsymbol{\rho}_{0, j} \in L^{1}((0, \infty))$ and $\tilde{\boldsymbol{\rho}}_{0, j} \in L^{1}((0, \infty))$ for any $j \in \mathcal{O}$ with initial datum for the Hamilton-Jacobi equations as in Definition 4.2. Then, we have the following estimate:

$$
\left\|\mathbf{V}_{j}^{\text {out }}[\mathbf{F}]-\tilde{\mathbf{V}}_{j}^{\text {out }}[\mathbf{F}]_{j}\right\|_{C([0, T])} \leq\left\|\boldsymbol{\rho}_{0, j}-\tilde{\boldsymbol{\rho}}_{0, j}\right\|_{L^{1}((0, \infty))}
$$

Proof.

(1) Let $t \in[0, T]$ and $i \in \mathcal{I}$ and let us first assume that the dominating terms in the solution formula are in both cases stemming from the initial datum. Let us assume that the maximum of the first term is attained at $y^{1} \in \mathbb{R}_{\leq 0}$ and for the second term at $y^{2} \in \mathbb{R}_{\leq 0}$. Then, we obtain the two-sided estimate

$$
\begin{aligned}
& \max _{y \in \mathbb{R}_{\leq 0}}\left\{\mathbf{V}_{0, i}^{\mathrm{in}}(y)+t \mathbf{f}_{i}^{*}\left(\frac{-y}{t}\right)\right\}-\max _{\tilde{y} \in \mathbb{R}_{\leq 0}}\left\{\tilde{\mathbf{V}}_{0, i}^{\mathrm{in}}(\tilde{y})+t \mathbf{f}_{i}^{*}\left(\frac{-\tilde{y}}{t}\right)\right\} \\
& \leq\left\{\mathbf{V}_{0, i}^{\mathrm{in}}\left(y^{1}\right)+t \mathbf{f}_{i}^{*}\left(\frac{-y^{1}}{t}\right)\right\}-\max _{\tilde{y} \in \mathbb{R}_{\leq 0}}\left\{\tilde{\mathbf{V}}_{0, i}^{\mathrm{in}}(\tilde{y})+t \mathbf{f}_{i}^{*}\left(\frac{-\tilde{y}}{t}\right)\right\} \\
& \leq\left|\mathbf{V}_{0, i}^{\mathrm{in}}\left(y^{1}\right)-\tilde{\mathbf{V}}_{0, i}^{\mathrm{in}}\left(y^{1}\right)\right| \leq \int_{-\infty}^{y^{1}}\left|\boldsymbol{\rho}_{0, i}(s)-\tilde{\boldsymbol{\rho}}_{0, i}(s)\right| \mathrm{d} s \leq\left\|\boldsymbol{\rho}_{0, i}-\tilde{\boldsymbol{\rho}}_{0, i}\right\|_{L^{1}((-\infty, 0))}
\end{aligned}
$$

and

$$
\begin{aligned}
& \max _{y \in \mathbb{R}_{\leq 0}}\left\{\mathbf{V}_{0, i}^{\text {in }}(y)+t \mathbf{f}_{i}^{*}\left(\frac{-y}{t}\right)\right\}-\max _{\tilde{y} \in \mathbb{R}_{\leq 0}}\left\{\tilde{\mathbf{V}}_{0, i}^{\text {in }}(\tilde{y})+t \mathbf{f}_{i}^{*}\left(\frac{-\tilde{y}}{t}\right)\right\} \\
& \geq \max _{y \in \mathbb{R}_{\leq 0}}\left\{\mathbf{V}_{0, i}^{\text {in }}(y)+t \mathbf{f}_{i}^{*}\left(\frac{-y}{t}\right)\right\}-\left\{\tilde{\mathbf{V}}_{0, i}^{\text {in }}\left(y^{2}\right)+t \mathbf{f}_{i}^{*}\left(\frac{-y^{2}}{t}\right)\right\}
\end{aligned}
$$




$$
\geq-\left\|\boldsymbol{\rho}_{0, i}-\tilde{\boldsymbol{\rho}}_{0, i}\right\|_{L^{1}((-\infty, 0))} .
$$

Since the estimates are uniform in $t$, the claim follows. Concentrating on the case where the solution is a function of the boundary datum we obtain similarly by picking specific $t_{1}, t_{2}, y \in[0, T]^{2} \times \mathbb{R}_{\leq 0}$ and by a similar argumentation as above

$$
\begin{aligned}
& \mid \max _{\substack{y \in \mathbb{R}_{\leq 0} \\
0 \leq t_{2} \leq t_{1} \leq t}}\left\{\mathbf{V}_{0, i}^{\text {in }}(y)+t_{2} \mathbf{f}_{i}^{*}\left(\frac{-y}{t_{2}}\right)-\left(t-t_{1}\right) \mathbf{f}_{i}^{\max }-\int_{t_{2}}^{t_{1}} \mathbf{h}_{i}(s) \mathrm{d} s\right\} \\
& \quad-\max _{\substack{y \in \mathbb{R}^{\leq 0} \\
0 \leq t_{2} \leq t_{1} \leq t}}\left\{\tilde{\mathbf{V}}_{0, i}^{\text {in }}(y)+t_{2} \mathbf{f}_{i}^{*}\left(\frac{-y}{t_{2}}\right)-\left(t-t_{1}\right) \mathbf{f}_{i}^{\max }-\int_{t_{2}}^{t_{1}} \mathbf{h}_{i}(s) \mathrm{d} s\right\} \mid \\
& \leq\left\|\boldsymbol{\rho}_{0, i}-\tilde{\boldsymbol{\rho}}_{0, i}\right\|_{L^{1}((-\infty, 0)) .}
\end{aligned}
$$

The other two mixed cases can be handled from those two recalling Lemma 4.5.

(2) Let $t>0$ and $j \in \mathcal{O}$, we obtain

$$
\begin{aligned}
& \mathbf{F}_{j}[\mathbf{V}](t)-\tilde{\mathbf{F}}_{j}[\mathbf{V}](t)=\mathbf{q}_{0, j}-\tilde{\mathbf{q}}_{0, j}-\sum_{i \in \mathcal{I}} \int_{0}^{t} \mathbf{V}_{i, s}(s) \boldsymbol{\theta}_{i, j}(s) \mathrm{d} s+\sum_{i \in \mathcal{I}} \int_{0}^{t} \mathbf{V}_{i, s}(s) \tilde{\boldsymbol{\theta}}_{i, j}(s) \mathrm{d} s \\
&=\mathbf{q}_{0, j}-\tilde{\mathbf{q}}_{0, j}+\sum_{i \in \mathcal{I}} \int_{0}^{t} \mathbf{V}_{i, s}(s)\left(\tilde{\boldsymbol{\theta}}_{i, j}(s)-\boldsymbol{\theta}_{i, j}(s)\right) \mathrm{d} s \\
&\left\|\mathbf{F}_{j}[\mathbf{V}]-\tilde{\mathbf{F}}_{j}[\mathbf{V}]\right\|_{C([0, T])} \leq\left|\mathbf{q}_{0, j}-\tilde{\mathbf{q}}_{0, j}\right|+\sum_{i \in \mathcal{I}} \mathbf{f}_{i}^{\max }\left\|\boldsymbol{\theta}_{i, j}-\tilde{\boldsymbol{\theta}}_{i, j}\right\|_{L^{1}((0, T)),}
\end{aligned}
$$

where the last inequality follows by the uniform Lipschitz-bound on $\mathbf{V}$.

(3) The proof follows likewise to the argument in Item 1.

The previously stated Lemma 5.1 together with Proposition 4.1 are the key ingredients for the following stability result in Theorem 5.1, which guarantees that, under small changes of the involved input datum, the solution, here the queue, can only have small limited variations.

THEOREM 5.1 (Stability of the queue). Let the framework in Section 4 and $T \in \mathbb{R}_{>0}$ be given. Assume that $\mathbf{q}_{0}, \tilde{\mathbf{q}}_{0} \in \mathbb{R}_{>0}^{|\mathcal{O}|}$ with $\left\|\mathbf{q}_{0}\right\|_{1},\left\|\tilde{\mathbf{q}}_{0}\right\|_{1} \leq M$. Assume that in addition $\boldsymbol{\rho}_{0, i}, \tilde{\boldsymbol{\rho}}_{0, i} \in L^{\infty}((-\infty, 0)) \cap L^{1}((-\infty, 0))$ with $0 \leq \boldsymbol{\rho}_{0, i} \leq \boldsymbol{\rho}_{i}^{\max }, \quad 0 \leq \tilde{\boldsymbol{\rho}}_{0, i} \leq \boldsymbol{\rho}_{i}^{\max }, \quad i \in \mathcal{I}$ and $\boldsymbol{\rho}_{0, j}, \tilde{\boldsymbol{\rho}}_{0, j} \in L^{\infty}((0, \infty)) \cap L^{1}((0, \infty))$ with $0 \leq \boldsymbol{\rho}_{0, j} \leq \boldsymbol{\rho}_{j}^{\max }, \quad 0 \leq \tilde{\boldsymbol{\rho}}_{0, j} \leq \boldsymbol{\rho}_{j}^{\max }, \quad j \in \mathcal{O}$ be given as well as two routings $\boldsymbol{\theta}, \tilde{\boldsymbol{\theta}} \in B V\left((0, T) ; \mathbb{R}_{\geq 0}^{|\mathcal{O}| \times|\mathcal{I}|}\right)$ with $\sum_{j \in \mathcal{O}} \boldsymbol{\theta}_{i, j}(t)=1=$ $\sum_{j \in \mathcal{O}} \tilde{\boldsymbol{\theta}}_{i, j}(t) \forall t \in[0, T]$ almost everywhere. Let $\mathbf{q}, \tilde{\mathbf{q}}$ be the corresponding solutions, the following stability result/Lipschitz continuity holds:

$$
\begin{aligned}
& \|\mathbf{q}-\tilde{\mathbf{q}}\|_{C\left([0, T] ; \mathbb{R}^{|\mathcal{O}|}\right)} \\
& \leq\left(2\left|\mathbf{q}_{0}-\tilde{\mathbf{q}}_{0}\right|_{1}+2 \sum_{(i, j) \in \mathcal{I} \times \mathcal{O}} \mathbf{f}_{i, j}^{\max }\left\|\boldsymbol{\theta}_{i, j}-\tilde{\boldsymbol{\theta}}_{i, j}\right\|_{L^{1}((0, t))}+\left\|\boldsymbol{\rho}_{0}-\tilde{\boldsymbol{\rho}}_{0}\right\|_{\left.L^{1}((0, \infty) ; \mathbb{R}|\mathcal{O}|)\right)}\right. \\
& \left.+2 \sum_{i, j \in \mathcal{I} \times \mathcal{O}}\left(\left\|\boldsymbol{\theta}_{i, j}\right\|_{T V((0, T))}+1\right)\left(\left\|\boldsymbol{\rho}_{0, i}-\tilde{\boldsymbol{\rho}}_{0, i}\right\|_{L^{1}((-\infty, 0))}\right)\right) \\
& \quad \cdot \exp \left(2 \sum_{(i, j) \in \mathcal{I} \times \mathcal{O}}\left(\left\|\boldsymbol{\theta}_{i, j}\right\|_{T V((0, T))}+1\right)\left\|\mathbf{c}_{i}\right\|_{L^{\infty}((0, T))} T\right)
\end{aligned}
$$


Proof. Let us first mention that $\mathbf{q}, \tilde{\mathbf{q}}$ exist and are unique due to Theorem 4.3. Thus, we can directly concentrate on the fixed-point mapping Lemma 4.4 which is satisfied by $\mathbf{q}, \tilde{\mathbf{q}}$, and indicate the involved functions dependent on the ' $\sim$ ' datum by the corresponding ' $\sim$ '. Then, we obtain for $t \in[0, T]$ and $j \in \mathcal{O}$

$$
\begin{aligned}
& \left|\mathbf{q}_{j}(t)-\tilde{\mathbf{q}}_{j}(t)\right| \\
& =\mid \boldsymbol{\Lambda}_{j}\left[\mathbf{F} \circ \mathbf{V}^{\text {in }} \circ \mathbf{h} \circ \mathbf{q}, \mathbf{V}^{\text {out }} \circ \mathbf{F} \circ \mathbf{V}^{\text {in }} \circ \mathbf{h} \circ \mathbf{q}\right](t) \\
& \quad-\boldsymbol{\Lambda}_{j}\left[\tilde{\mathbf{F}} \circ \tilde{\mathbf{V}}^{\text {in }} \circ \mathbf{h} \circ \tilde{\mathbf{q}}, \tilde{\mathbf{V}}^{\text {out }} \circ \tilde{\mathbf{F}} \circ \tilde{\mathbf{V}}^{\text {in }} \circ \mathbf{h} \circ \tilde{\mathbf{q}}\right](t) \mid
\end{aligned}
$$

plugging in the definition of $\boldsymbol{\Lambda}$ in Definition 4.3

$$
\begin{aligned}
= & \mid \mathbf{F}_{j}\left[\mathbf{V}^{\text {in }} \circ \mathbf{h} \circ \mathbf{q}\right](t)-\tilde{\mathbf{F}}_{j}\left[\tilde{\mathbf{V}}^{\text {in }} \circ \mathbf{h} \circ \tilde{\mathbf{q}}\right](t) \\
& +\mathbf{V}_{j}^{\text {out }}\left[\mathbf{F} \circ \mathbf{V}^{\text {in }} \circ \mathbf{h} \circ \mathbf{q}\right](t)-\tilde{\mathbf{V}}_{j}^{\text {out }}\left[\tilde{\mathbf{F}} \circ \tilde{\mathbf{V}}^{\text {in }} \circ \mathbf{h} \circ \tilde{\mathbf{q}}\right](t) \mid \\
\leq & \left|\mathbf{F}_{j}\left[\mathbf{V}^{\text {in }} \circ \mathbf{h} \circ \mathbf{q}\right](t)-\tilde{\mathbf{F}}_{j}\left[\tilde{\mathbf{V}}^{\text {in }} \circ \mathbf{h} \circ \tilde{\mathbf{q}}\right](t)\right| \\
& +\left|\mathbf{V}_{j}^{\text {out }}\left[\mathbf{F} \circ \mathbf{V}^{\text {in }} \circ \mathbf{h} \circ \mathbf{q}\right](t)-\mathbf{V}_{j}^{\text {out }}\left[\tilde{\mathbf{F}} \circ \tilde{\mathbf{V}}^{\text {in }} \circ \mathbf{h} \circ \tilde{\mathbf{q}}\right](t)\right| \\
& +\left|\mathbf{V}_{j}^{\text {out }}\left[\tilde{\mathbf{F}} \circ \tilde{\mathbf{V}}^{\text {in }} \circ \mathbf{h} \circ \tilde{\mathbf{q}}\right](t)-\tilde{\mathbf{V}}_{j}^{\text {out }}\left[\tilde{\mathbf{F}} \circ \tilde{\mathbf{V}}^{\text {in }} \circ \mathbf{h} \circ \tilde{\mathbf{q}}\right](t)\right|
\end{aligned}
$$

applying on the latter terms Lemma 5.1 and Proposition 4.1

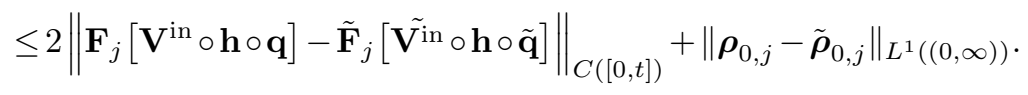

Concentrating on the first term, we have

$$
\begin{aligned}
& \| \mathbf{F}_{j}\left[\mathbf{V}^{\text {in }} \circ \mathbf{h} \circ \mathbf{q}\right]-\tilde{\mathbf{F}}_{j}\left[\tilde{\left.\mathbf{V}^{\text {in }} \circ \mathbf{h} \circ \tilde{\mathbf{q}}\right]} \|_{C([0, t])}\right. \\
& \leq\left\|\mathbf{F}_{j}\left[\mathbf{V}^{\text {in }} \circ \mathbf{h} \circ \mathbf{q}\right]-\tilde{\mathbf{F}}_{j}\left[\mathbf{V}^{\text {in }} \circ \mathbf{h} \circ \mathbf{q}\right]\right\|_{C([0, t])}+\| \tilde{\mathbf{F}}_{j}\left[\mathbf{V}^{\text {in }} \circ \mathbf{h} \circ \mathbf{q}\right]-\tilde{\mathbf{F}}_{j}\left[\tilde{\left.\mathbf{V}^{\text {in }} \circ \mathbf{h} \circ \tilde{\mathbf{q}}\right]} \|_{C([0, t])}\right.
\end{aligned}
$$

using again Lemma 5.1 and Proposition 4.1 we obtain

$$
\begin{aligned}
\leq & \left|\mathbf{q}_{0, j}-\tilde{\mathbf{q}}_{0, j}\right|+\sum_{i \in \mathcal{I}} \mathbf{f}_{i}^{\max }\left\|\boldsymbol{\theta}_{i, j}-\tilde{\boldsymbol{\theta}}_{i, j}\right\|_{L^{1}((0, t))} \\
& +\sum_{i \in \mathcal{I}}\left(\left\|\boldsymbol{\theta}_{i, j}\right\|_{T V((0, T))}+1\right)\left\|\mathbf{V}_{i}^{\text {in }}[\mathbf{h} \circ \mathbf{q}]-\tilde{\mathbf{V}}_{i}^{\text {in }}[\mathbf{h} \circ \tilde{\mathbf{q}}]\right\| \|_{C([0, t])} .
\end{aligned}
$$

Finally, it remains to estimate the latter term and we obtain for this term again using Lemma 5.1, Proposition 4.1

$$
\begin{aligned}
& \left\|\mathbf{V}_{i}^{\text {in }}[\mathbf{h} \circ \mathbf{q}]-\tilde{\mathbf{V}}_{i}^{\text {in }}[\mathbf{h} \circ \tilde{\mathbf{q}}]\right\|_{C([0, t])} \\
& \leq\left\|\mathbf{V}_{i}^{\text {in }}[\mathbf{h} \circ \mathbf{q}]-\tilde{\mathbf{V}}_{i}^{\text {in }}[\mathbf{h} \circ \mathbf{q}]\right\|_{C([0, t])}+\left\|\tilde{\mathbf{V}}_{i}^{\text {in }}[\mathbf{h} \circ \mathbf{q}]-\tilde{\mathbf{V}}^{\text {in }}[\mathbf{h} \circ \tilde{\mathbf{q}}]\right\|_{C([0, t])} \\
& \leq\left\|\boldsymbol{\rho}_{0, i}-\tilde{\boldsymbol{\rho}}_{0, i}\right\|_{L^{1}((-\infty, 0))}+\left\|\mathbf{h}_{i}[\mathbf{q}]-\mathbf{h}_{i}[\tilde{\mathbf{q}}]\right\|_{L^{1}((0, t))} \\
& \leq\left\|\boldsymbol{\rho}_{0, i}-\tilde{\boldsymbol{\rho}}_{0, i}\right\|_{L^{1}((-\infty, 0))}+\left\|\mathbf{c}_{i}\right\|_{L^{\infty}((0, t))} \sum_{j \in \mathcal{O}}\left\|\mathbf{q}_{j}-\tilde{\mathbf{q}}_{j}\right\|_{L^{1}((0, t))} .
\end{aligned}
$$


Combining all the upper estimates and summarizing, we then have for $t \in[0, T]$

$$
\begin{aligned}
& \left|\mathbf{q}_{j}(t)-\tilde{\mathbf{q}}_{j}(t)\right| \\
& \leq 2\left|\mathbf{q}_{0, j}-\tilde{\mathbf{q}}_{0, j}\right|+2 \sum_{i \in \mathcal{I}} \mathbf{f}_{i}^{\max }\left\|\boldsymbol{\theta}_{i, j}-\tilde{\boldsymbol{\theta}}_{i, j}\right\|_{L^{1}((0, t))}+\left\|\boldsymbol{\rho}_{0, j}-\tilde{\boldsymbol{\rho}}_{0, j}\right\|_{L^{1}((0, \infty))} \\
& +2 \sum_{i \in \mathcal{I}}\left(\left\|\boldsymbol{\theta}_{i, j}\right\|_{T V((0, T))}+1\right)\left(\left\|\boldsymbol{\rho}_{0, i}-\tilde{\boldsymbol{\rho}}_{0, i}\right\|_{L^{1}((-\infty, 0))}+\left\|\mathbf{c}_{i}\right\|_{L^{\infty}((0, t))} \sum_{j \in \mathcal{O}}\left\|\mathbf{q}_{j}-\tilde{\mathbf{q}}_{j}\right\|_{L^{1}((0, t))}\right)
\end{aligned}
$$

Summing over all $j$ we obtain

$$
\begin{aligned}
\sum_{j \in \mathcal{O}}\left|\mathbf{q}_{j}(t)-\tilde{\mathbf{q}}_{j}(t)\right| \leq & 2\left|\mathbf{q}_{0}-\tilde{\mathbf{q}}_{0}\right|_{1}+2 \sum_{i, j \in \mathcal{I} \times \mathcal{O}} \mathbf{f}_{i}^{\max }\left\|\boldsymbol{\theta}_{i, j}-\tilde{\boldsymbol{\theta}}_{i, j}\right\|_{L^{1}((0, t))} \\
& +\left\|\boldsymbol{\rho}_{0}-\tilde{\boldsymbol{\rho}}_{0}\right\|_{\left.L^{1}\left((0, \infty) ; \mathbb{R}^{|\mathcal{O}|}\right)\right)}+2 \sum_{(i, j) \in \mathcal{I} \times \mathcal{O}}\left(\left\|\boldsymbol{\theta}_{i, j}\right\|_{T V((0, T))}+1\right) \\
& \cdot\left(\left\|\boldsymbol{\rho}_{0, i}-\tilde{\boldsymbol{\rho}}_{0, i}\right\|_{L^{1}((-\infty, 0))}+\left\|\mathbf{c}_{i}\right\|_{L^{\infty}((0, t))} \sum_{j \in \mathcal{O}}\left\|\mathbf{q}_{j}-\tilde{\mathbf{q}}_{j}\right\|_{L^{1}((0, t))}\right) .
\end{aligned}
$$

We can now apply Gronwall's inequality in [36, Theorem 1.3.1] to obtain for any $t \in[0, T]$ :

$$
\begin{aligned}
& \sum_{j \in \mathcal{O}}\left|\mathbf{q}_{j}(t)-\tilde{\mathbf{q}}_{j}(t)\right| \\
& \leq\left(2\left|\mathbf{q}_{0}-\tilde{\mathbf{q}}_{0}\right|_{1}+2 \sum_{i, j \in \mathcal{I} \times \mathcal{O}} \mathbf{f}_{i}^{\max }\left\|\boldsymbol{\theta}_{i, j}-\tilde{\boldsymbol{\theta}}_{i, j}\right\|_{L^{1}((0, t))}\right. \\
& \quad+\left\|\boldsymbol{\rho}_{0}-\tilde{\boldsymbol{\rho}}_{0}\right\|_{\left.L^{1}((0, \infty) ; \mathbb{R}|\mathcal{O}|)\right)}+2 \sum_{i, j \in \mathcal{I} \times \mathcal{O}}\left(\left\|\boldsymbol{\theta}_{i, j}\right\|_{T V((0, T))}+1\right) \\
& \left.\quad \cdot\left(\left\|\boldsymbol{\rho}_{0, i}-\tilde{\boldsymbol{\rho}}_{0, i}\right\|_{L^{1}((-\infty, 0))}\right)\right) \exp \left(2 \sum_{i, j \in \mathcal{I} \times \mathcal{O}}\left(\left\|\boldsymbol{\theta}_{i, j}\right\|_{T V((0, T))}+1\right)\left\|\mathbf{c}_{i}\right\|_{L^{\infty}((0, t))} t\right) .
\end{aligned}
$$

Taking the supremum over all $t \in[0, T]$, we obtain Lipschitz-continuity of the queues w.r.t. initial, datum, initial buffer load and routing as pointed out in the statement of the theorem.

\section{Optimal Routing}

For now we have not discussed how we would actually determine the optimal routing $\boldsymbol{\theta}$ i.e. the optimal trajectory of vehicles, for specific traffic information patterns. One way to approach this - assuming full information of inflow on the network - is to consider a minimization problem where we would minimize the queue size at a specific node following the basic idea that the smaller the queue size is the lesser the congestion becomes. For the single destination case we consider in this article we can use the previously stated Theorem 5.1 to obtain existence of a minimizer. This is detailed below.

THEOREM 6.1 (Optimal Routing - multi queue buffer). Let a junction with $|\mathcal{I}| \in \mathbb{N}_{\geq 1}$ incoming and $|\mathcal{O}| \in \mathbb{N}_{\geq 1}$ outgoing links be given and assume the dynamics hold in the sense of Definition 3.1. Then, for any $p \in[1, \infty]$ and $K \in \mathbb{R}_{\geq 0}$ the two minimization problems

$$
\bullet \min _{\boldsymbol{\theta} \in \boldsymbol{\Theta}_{K}} \sum_{j \in \mathcal{O}}\left\|\mathbf{q}_{j}[\boldsymbol{\theta}]\right\|_{L^{p}((0, T))} \quad \bullet \min _{\boldsymbol{\theta} \in \boldsymbol{\Theta}} \sum_{j \in \mathcal{O}}\left\|\mathbf{q}_{j}[\boldsymbol{\theta}]\right\|_{L^{p}((0, T))}+\sum_{(i, j) \in \mathcal{I} \times \mathcal{O}}\left\|\boldsymbol{\theta}_{i, j}\right\|_{T V((0, T))}
$$


subject to the dynamics in Definition 3.1 with

$$
\begin{aligned}
\boldsymbol{\Theta} & :=\left\{\boldsymbol{\theta} \in B V\left((0, T) ; \mathbb{R}^{|\mathcal{I}| \times|\mathcal{O}|}\right): 0 \leq \boldsymbol{\theta}_{i, j}(t) \leq 1 \wedge \sum_{j \in \mathcal{O}} \boldsymbol{\theta}_{i, j}(t)=1 \forall t \in[0, T] \text { a.e. } \forall(i, j) \in \mathcal{I} \times \mathcal{O}\right\} \\
\boldsymbol{\Theta}_{K} & :=\left\{\boldsymbol{\theta} \in \boldsymbol{\Theta}:\|\boldsymbol{\theta}\|_{T V((0, T) ; \mathbb{R}|\mathcal{I}| \times|\mathcal{O}|)} \leq K\right\}
\end{aligned}
$$

admit a solution. We denote the dependency of $\mathbf{q}$ w.r.t. assumed routing $\boldsymbol{\theta}$ by $\mathbf{q}[\boldsymbol{\theta}]$.

Proof. We start with proving the claim for the first objective function. Due to the fact that the objective functions are both bounded from below, there exists a sequence $\left(\boldsymbol{\theta}_{l}\right)_{l \in \mathbb{N}} \subseteq \boldsymbol{\Theta}_{K}$ so that

$$
\lim _{l \rightarrow \infty} \sum_{j \in \mathcal{O}}\left\|\mathbf{q}_{j}\left[\boldsymbol{\theta}_{l}\right]\right\|_{L^{p}((0, T))}=\inf _{\boldsymbol{\theta} \in \boldsymbol{\Theta}} \sum_{j \in \mathcal{O}}\left\|\mathbf{q}_{j}[\boldsymbol{\theta}]\right\|_{L^{p}((0, T))} \geq 0
$$

Since $B V((0, T)) \stackrel{\text { c }}{\hookrightarrow} L^{p}((0, T))$, i.e. $B V$ is for space dimension 1 compactly embedded into $L^{p}((0, T)) \forall p \in[1, \infty)$ (see [34, Theorem 13.32 (Rellich Kondrachov), Theorem 13.35 (Compactness)] we can pick a subsequence $\left(\boldsymbol{\theta}_{l_{m}}\right)_{m \in \mathbb{N}} \subset \boldsymbol{\Theta}_{K}$ such that

$$
\exists \boldsymbol{\theta}^{*} \in \boldsymbol{\Theta}_{K}: \lim _{m \rightarrow \infty}\left\|\boldsymbol{\theta}^{*}-\boldsymbol{\theta}_{l_{m}}\right\|_{L^{1}\left((0, T) ; \mathbb{R}^{|\mathcal{I}| \times|\mathcal{O}|)}\right.}=0
$$

(see also [4, Proposition 3.13, Theorem 3.23]). Due to Theorem 5.1 we then know that

$$
\lim _{m \rightarrow \infty}\left\|\mathbf{q}\left[\boldsymbol{\theta}^{*}\right]-\mathbf{q}\left[\boldsymbol{\theta}_{l_{m}}\right]\right\|_{C\left([0, T] ; \mathbb{R}^{|\mathcal{O}|}\right)}=0
$$

so that in particular due to $C([0, T]) \hookrightarrow L^{p}((0, T))$ the objective function also strongly converges. This proves the claim for the first minimization problem.

Almost the same argumentation can be made for the second objective noticing that due to the $B V$ norm in the objective function we have, for any minimizing sequence $\left(\boldsymbol{\theta}_{k}\right)_{k \in \mathbb{N}} \subset \boldsymbol{\Theta}$, that there exists $C \in \mathbb{R}_{>0}$ so that $\sup _{k \in \mathbb{N}} \sum_{(i, j) \in \mathcal{I} \times \mathcal{O}}\left\|\boldsymbol{\theta}_{i, j}\right\|_{B V((0, T))} \leq C$ so that we can follow the same reasoning as for the first objective function.

REMARK 6.1 (No uniqueness of the Optimal Control problem and different objective functions). It is not surprising that one does not obtain a uniqueness result for the previously mentioned optimal control problem for the first objective function. For that consider zero initial datum for the incoming and outgoing edges, and assume that $\boldsymbol{q}_{0}=\boldsymbol{O}$. Then, obviously every routing results in a queuing size of zero as long as the routing respects the bound $K \in \mathbb{R}_{\geq 0}$. The result also holds if the initial datum in the incoming and outgoing links is sufficiently small, so no buffer is needed. A similar argument holds for the second objective. Even though any change in routing over time is penalized by the $T V$ semi-norm for sufficiently small initial datum, any constant routing would produce a queueing size of zero.

One can replace the objective functions used in Theorem 6.1 with more general ones. The only requirement is the boundedness from below and the lower semicontinuity of the used norm.

\section{Implementation of the model on general road networks}

This section implements our framework on a realistic road network, which contains several intersections and finite-length links. The classical argument used in the literature on conservation laws on road networks is that the information has finite propagation speed. We explore how this argument is detailed mathematically. The general idea is the following: Consider a link of length $L \in \mathbb{R}_{>0}$, connecting intersections $v_{1}$ and $v_{2}$. We 
need to prove that for sufficiently small time, only a neighborhood of the link around $v_{1}$ can be affected by the information exiting this intersection, and the same for $v_{2}$. After this, we will know that outside this neighborhood, the solution of Hamilton-Jacobi equations on this link can be solved only considering initial datum. This is a classical argument for conservation laws and we now detail this argument for the considered class of Hamilton-Jacobi equations. We then need to prove that, for a given finite-time horizon, there exists a distance $d$, such that if $|x| \geq d$, the solution formula always selects information emanating from the initial datum, thus reducing the IBVP problem to a Cauchy problem. We prove the following lemma for an incoming link.

LEMMA 7.1 (Finite propagation speed of information in Hamilton-Jacobi solutions). For $t \in[0, T]$ let $x \leq 0$ such that $x \leq \mathbf{f}_{i}^{\prime}\left(\boldsymbol{\rho}_{i}^{\max }\right) t$. Then

$$
\begin{aligned}
& \overline{\mathbf{V}}_{i}^{\text {in }}(t, x)=\max \left\{\begin{array}{c}
\max _{y \in \mathbb{R}_{\leq 0}}\left\{\mathbf{V}_{0, i}^{\text {in }}(y)+t \mathbf{f}_{i}^{*}\left(\frac{x-y}{t}\right)\right\}, \\
\left.\max _{\substack{y \in \mathbb{R}_{\leq 0} \\
0 \leq t_{2} \leq t_{1} \leq t}}\left\{\mathbf{V}_{0, i}^{\text {in }}(y)+t_{2} \mathbf{f}_{i}^{*}\left(\frac{-y}{t_{2}}\right)+\left(t-t_{1}\right) \mathbf{f}_{i}^{*}\left(\frac{x}{t-t_{1}}\right)-\int_{t_{2}}^{t_{1}} \mathbf{h}_{i}(s) \mathrm{d} s\right\}\right\}
\end{array}\right. \\
& =\max _{y \in \mathbb{R}_{\leq 0}}\left\{\mathbf{V}_{0, i}^{\mathrm{in}}(y)+t \mathbf{f}_{i}^{*}\left(\frac{x-y}{t}\right)\right\} .
\end{aligned}
$$

Proof. We know that for any $x \in \mathbb{R}$

$$
f^{*}(x):=\inf _{u \in\left[0, \rho^{\max }\right]}\{u x-f(u)\}, x \in \operatorname{Dom}\left(f^{*}\right) .
$$

Let $i \in \mathcal{I}$. Assume that $x<\mathbf{f}_{i}^{\prime}\left(\boldsymbol{\rho}_{i}^{\max }\right)$. Then for any $u \in\left[0, \boldsymbol{\rho}_{i}^{\max }\right.$ [ we have

$$
\begin{gathered}
x<\frac{\mathbf{f}_{i}(u)-\mathbf{f}_{i}\left(\boldsymbol{\rho}_{i}^{\max }\right)}{u-\boldsymbol{\rho}_{i}^{\max }} \\
u x-\mathbf{f}_{i}(u)>\boldsymbol{\rho}_{i}^{\max } x .
\end{gathered}
$$

In addition we know that by picking as argument $u=\boldsymbol{\rho}_{i}^{\max }$ in the infimum we get $\mathbf{f}_{i}^{*}(x) \leq \boldsymbol{\rho}_{i}^{\max } x$. Now let us fix $t \in[0, T], \mathbf{h}_{i} \in \operatorname{Lip}([0, T])$ and look at

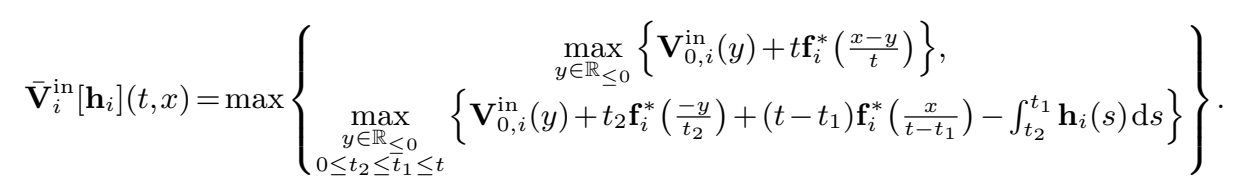

For $t \in[0, T]$, let $x \leq 0$ such that $\frac{x}{t} \leq \mathbf{f}_{i}^{\prime}\left(\boldsymbol{\rho}_{i}^{\max }\right)$. Then for any $0<t_{1}<t$, we have $\frac{x}{t-t_{1}} \leq \frac{x}{t} \leq \mathbf{f}_{i}^{\prime}\left(\boldsymbol{\rho}_{i}^{\max }\right)$.

In addition, for any $t_{1} \leq t$ we have $\left(t-t_{1}\right) \mathbf{f}_{i}^{*}\left(\frac{x}{t-t_{1}}\right) \leq x \boldsymbol{\rho}_{i}^{\max }$. Thus

$$
\begin{aligned}
& \max _{\substack{y \in \mathbb{R}_{\leq 0} \leq t \\
0 \leq t_{2} \leq t_{1} \leq t}}\left\{\mathbf{V}_{0, i}^{\text {in }}(y)+t_{2} \mathbf{f}_{i}^{*}\left(\frac{-y}{t_{2}}\right)+\left(t-t_{1}\right) \mathbf{f}_{i}^{*}\left(\frac{x}{t-t_{1}}\right)-\int_{t_{2}}^{t_{1}} \mathbf{h}_{i}(s) \mathrm{d} s\right\} \\
& \leq x \boldsymbol{\rho}_{i}^{\max }+\max _{\substack{y \in \mathbb{R}_{\leq} \leq 0 \\
0 \leq t_{2} \leq t}}\left\{\mathbf{V}_{0, i}^{\text {in }}(y)+t_{2} \mathbf{f}_{i}^{*}\left(\frac{-y}{t_{2}}\right)\right\},
\end{aligned}
$$

assuming that $\mathbf{h}_{i} \geq 0$. We need to prove now that

$$
x \boldsymbol{\rho}_{i}^{\max }+\max _{\substack{y \in \mathbb{R} \leq 0 \\ 0 \leq t_{2} \leq t}}\left\{\mathbf{V}_{0, i}^{\mathrm{in}}(y)+t_{2} \mathbf{f}_{i}^{*}\left(\frac{-y}{t_{2}}\right)\right\} \leq \max _{y \in \mathbb{R}_{\leq 0}}\left\{\mathbf{V}_{0, i}^{\mathrm{in}}(y)+t \mathbf{f}_{i}^{*}\left(\frac{x-y}{t}\right)\right\} .
$$


Picking $\bar{y}$ and $\bar{t}_{2}$ optimal in the left maximum, we need to look at $\bar{t}_{2} \mathbf{f}_{i}^{*}\left(\frac{-\bar{y}}{\bar{t}_{2}}\right)-$ $t \mathbf{f}_{i}^{*}\left(\frac{x-y}{t}\right)$ and show " $\leq 0$ ".

$$
\begin{aligned}
& x \boldsymbol{\rho}_{i}^{\max }+\max _{\substack{y \in \mathbb{R}_{\leq 0} \\
0 \leq t_{2} \leq t}}\left\{\mathbf{V}_{0, i}^{\mathrm{in}}(y)+t_{2} \mathbf{f}_{i}^{*}\left(\frac{-y}{t_{2}}\right)\right\}-\max _{y \in \mathbb{R}_{\leq 0}}\left\{\mathbf{V}_{0, i}^{\text {in }}(y)+t \mathbf{f}_{i}^{*}\left(\frac{x-y}{t}\right)\right\} \\
& \leq \underbrace{x \boldsymbol{\rho}_{i}^{\max }}_{\leq 0}+\underbrace{\bar{t}_{2} \mathbf{f}_{i}^{*}\left(\frac{-\bar{y}}{\bar{t}_{2}}\right)}_{\leq 0} \underbrace{-t \mathbf{f}_{i}^{*}\left(\frac{x-\bar{y}}{t}\right)}_{\geq 0} .
\end{aligned}
$$

- First, assume $\mathbf{f}_{i}^{\prime}\left(\boldsymbol{\rho}_{i}^{\max }\right) \leq \frac{x-\bar{y}}{t} \leq \mathbf{f}_{i}^{\prime}(0)$. Then $\mathbf{f}_{i}^{*}\left(\frac{x-\bar{y}}{t}\right)=u^{*} \frac{x-\bar{y}}{t}-\mathbf{f}_{i}\left(u^{*}\right)$ where $\mathbf{f}_{i}^{\prime}\left(u^{*}\right)=$ $\frac{x-\bar{y}}{t}$. By concavity of $\mathbf{f}_{i}$, the function $g:\left[0, \boldsymbol{\rho}_{i}^{\max }\right], u \rightarrow u \mathbf{f}_{i}^{\prime}(u)-u$ is decreasing, and can thus be lower bounded by $g\left(\boldsymbol{\rho}_{i}^{\max }\right)$. Then $\mathbf{f}_{i}^{*}\left(\frac{x-\bar{y}}{t}\right) \geq \boldsymbol{\rho}_{i}^{\max } \mathbf{f}_{i}^{\prime}\left(\boldsymbol{\rho}_{i}^{\max }\right)$ and

$$
x \boldsymbol{\rho}_{i}^{\max }+\bar{t}_{2} \mathbf{f}_{i}^{*}\left(\frac{-\bar{y}}{\bar{t}_{2}}\right)-t \mathbf{f}_{i}^{*}\left(\frac{x-\bar{y}}{t}\right) \leq\left(x-t \mathbf{f}_{i}^{\prime}\left(\boldsymbol{\rho}_{i}^{\max }\right)\right) \boldsymbol{\rho}_{i}^{\max } \leq 0 .
$$

- Now assume $\frac{x-\bar{y}}{t}>\mathbf{f}_{i}^{\prime}(0)$ or $\frac{x-\bar{y}}{t}<f^{\prime}\left(\boldsymbol{\rho}_{i}^{\max }\right)$. Then, if $\frac{x-\bar{y}}{t}-1 \geq 0$, the function $u \mapsto u \frac{x-\bar{y}}{t}-u$ is increasing on $\left[0, \boldsymbol{\rho}_{i}^{\max }\right]$, and thus $\mathbf{f}_{i}^{*}\left(\frac{x-\bar{y}}{t}\right)=0$ which provides the requested bound. Else, if $\frac{x-\bar{y}}{t}-1<0$, then the function $u \mapsto u \frac{x-\bar{y}}{t}-u$ is decreasing, and $\mathbf{f}_{i}^{*}\left(\frac{x-\bar{y}}{t}\right)=\boldsymbol{\rho}_{i}^{\max } \frac{x-\bar{y}}{t}$. Then

$$
\begin{aligned}
x \boldsymbol{\rho}_{i}^{\max }+\bar{t}_{2} \mathbf{f}_{i}^{*}\left(\frac{-\bar{y}}{t_{2}}\right)-t \mathbf{f}_{i}^{*}\left(\frac{x-\bar{y}}{t}\right) & =x \boldsymbol{\rho}_{i}^{\max }+\bar{t}_{2} \mathbf{f}_{i}^{*}\left(\frac{-\bar{y}}{\bar{t}_{2}}\right)-t \boldsymbol{\rho}_{i}^{\max } \frac{x-\bar{y}}{t} \\
& =\bar{y} \boldsymbol{\rho}_{i}^{\max }+\bar{t}_{2} \mathbf{f}_{i}^{*}\left(\frac{-\bar{y}}{\bar{t}_{2}}\right) \leq 0 .
\end{aligned}
$$

In all cases, we thus have that if $x-\mathbf{f}_{i}^{\prime}\left(\boldsymbol{\rho}_{i}^{\max }\right) t \leq 0$

$$
x \boldsymbol{\rho}_{i}^{\max }+\max _{\substack{y \in \mathbb{R}_{\leq 0} \\ 0 \leq t_{2} \leq t}}\left\{\mathbf{V}_{0, i}^{\mathrm{in}}(y)+t_{2} \mathbf{f}_{i}^{*}\left(\frac{-y}{t_{2}}\right)\right\} \leq \max _{y \in \mathbb{R}_{\leq 0}}\left\{\mathbf{V}_{0, i}^{\mathrm{in}}(y)+t \mathbf{f}_{i}^{*}\left(\frac{x-y}{t}\right)\right\} .
$$

This proves the "finite propagation speed argument", which claims that, if we are far enough from an intersection, the solution to the initial boundary-value problem is in fact the solution of the initial-value problem, because the information could not travel the distance and "reach" the location. This key argument allows the implementation of our framework to any "physical" road network. The same argument can be proved if one considers an outgoing link. In this case, the initial-boundary value problem can be reduced to an initial-value problem if $x-\mathbf{f}_{j}^{\prime}(0) t \geq 0, j \in \mathcal{O}$.

We consider a road network represented by a directed graph $G=(\mathcal{N}, \mathcal{L})$ containing $n$ nodes and $l$ finite-length links. For each link $l \in \mathcal{L}$, we denote its length by $L_{l}>0$. We then want to solve the following Cauchy problem on each link $l \in \mathcal{L}$.

$$
\begin{aligned}
\boldsymbol{\rho}_{l, t}(t, x)+\mathbf{f}_{l}\left(\boldsymbol{\rho}_{l}(t, x)\right)_{x} & =0, & (t, x) \in(0, T) \times\left(0, L_{l}\right), \\
\boldsymbol{\rho}_{l}(0, x) & =\boldsymbol{\rho}_{0, l}(x), & x \in\left(0, L_{l}\right) .
\end{aligned}
$$

The main idea of resolution is the following: For a small-enough time horizon, we can solve a fixed-point problem locally around each intersection, determining the solution locally on the incoming and outgoing links. The finite propagation speed of information ensures that the intersection will not be attained in this time-interval by information emanating from another junction. Outside of those neighborhoods, i.e. far away from intersection, we solve on the same time-interval an initial value problem. Finally we 
treat the solution as an initial datum and iterate. For each node $n \in \mathcal{N}$ Theorem 4.3 guarantees for each intersection, existence of a finite time-horizon $T_{n}$ on which the fixed point problem is well defined. We can then define $T_{\mathcal{N}}:=\min _{n \in \mathcal{N}} T_{n}$. In addition, we need to precise how we extend the initial value on semi-infinite links. Let us consider a given intersection $v$ and two links $(i, j) \in \mathcal{I}_{v} \times \mathcal{O}_{v}$. For the exiting link $j$ we can simply extend the initial datum $\boldsymbol{\rho}_{0, j}(x), x \in\left(0, L_{j}\right)$ by $\overline{\boldsymbol{\rho}}_{0, j}(x), x \geq 0$ such that

$$
\begin{aligned}
\overline{\boldsymbol{\rho}}_{0, j}(x) & = \begin{cases}\boldsymbol{\rho}_{0, j}(x) & \text { if } x \in\left(0, L_{j}\right) \\
0 & \text { else, }\end{cases} \\
\mathbf{V}_{0, j}^{\text {out }}(x) & =\int_{0}^{x} \overline{\boldsymbol{\rho}}_{0, j}(y) \mathrm{d} y, \quad x \in(0, \infty) .
\end{aligned}
$$

For the incoming link $i$ we need to define a space variable $\bar{x} \in(-\infty, 0)$. For $x \in\left(0, L_{i}\right)$ consider the change of variable $\bar{x}$ defined by $\bar{x}=x-L_{i}$. We then define

$$
\begin{aligned}
\overline{\boldsymbol{\rho}}_{0, i}(\bar{x}) & = \begin{cases}\boldsymbol{\rho}_{0, i}\left(\bar{x}+L_{i}\right) & \text { if } \bar{x} \in\left(-L_{i}, 0\right) \\
0 & \text { else, }\end{cases} \\
\mathbf{V}_{0, i}^{\text {in }}(\bar{x}) & =\int_{-\infty}^{\bar{x}} \overline{\boldsymbol{\rho}}_{0, i}(y) \mathrm{d} y \quad \bar{x} \in(-\infty, 0) .
\end{aligned}
$$

For each intersection we can then solve the fixed-point problem on $\left[0, T_{\mathcal{N}}\right]$ and obtain for all $n \in \mathcal{N}, j \in \mathcal{O}_{v}, t \in\left[0, T_{\mathcal{N}}\right]$ the solution $t \rightarrow \mathbf{q}_{j}^{n}(t)$.

Now that we have this solution, we explain how to obtain the solution to the inital Cauchy problem (7.2) on each link $l \in \mathcal{L}$.

Let us focus on one link $l$ connecting $v_{1}$ to $v_{2}$ whose space-coordinates can be indexed on $\left[0, L_{l}\right]$. By definition of $T_{\mathcal{N}}$ and application of Theorem 4.3 , the fixed-point problem is well posed for each intersection $v$ on $\left[0, T_{\mathcal{N}}\right]$. We know consider a new time-horizon $\mathrm{T}$, defined as $T=\min \left\{T_{\mathcal{N}}, \frac{L_{l}}{\mathbf{f}_{l}^{\prime}(0)-\mathbf{f}_{l}^{\prime}\left(\boldsymbol{\rho}_{l}^{\max }\right)}\right\}$, where $\frac{L_{l}}{\mathbf{f}_{l}^{\prime}(0)-\mathbf{f}_{l}^{\prime}\left(\boldsymbol{\rho}_{l}^{\max }\right)}$ represents the first instant at which information emanating from both intersections may interact. Then, for any $x \in\left[0, L_{l}\right]$, where $x$ denotes the distance from intersection $v_{1}$ and $-x+L_{l}$ denotes the distance to intersection $v_{2}$, we can define the solution in the following way:

- If $x<\mathbf{f}_{i}^{\prime}(0) T$, the point is in the neighborhood of the first intersection $v_{1}$ and the solution depends on the initial datum and the boundary condition imposed by $v_{1}$. The Hamilton Jacobi solution to the problem is $\overline{\mathbf{V}}_{l}^{\text {out }}(t, x)$ obtained from solving the fixed-point problem around $v_{1}$. This corresponds to zone A in Fig. 7.1.

- If $\left|x-L_{l}\right|<-\mathbf{f}_{i}^{\prime}\left(\boldsymbol{\rho}_{i}^{\max }\right) T, i \in \mathcal{I}$, the point is in the neighborhood of the second intersection $v_{2}$. Then the Hamilton Jacobi solution to the problem is $\overline{\mathbf{V}}_{l}^{\text {in }}\left(t, x-L_{l}\right)$ obtained from solving the fixed-point problem around $v_{2}$. This corresponds to zone C in Fig. 7.1.

- Else, the point is outside both neighborhoods, and can only be reached by information originating from the initial datum. This corresponds to zone B in Fig. 7.1. The solution $\overline{\mathbf{V}}_{l}(t, x)$ is defined as the solution of the initial value problem

$$
\begin{aligned}
\mathbf{v}_{l, t}(t, x)+\mathbf{f}_{l}\left(\mathbf{v}_{x}(t, x)\right) & =0 & & (t, x) \in(0, T) \times \mathbb{R}_{\geq 0} \\
\mathbf{v}(0, x) & =\mathbf{v}_{0, l}(x)=\int_{0}^{x} \boldsymbol{\rho}_{0, l}(y) d y & & x \in \mathbb{R}_{\geq 0}
\end{aligned}
$$

i.e. $\quad \overline{\mathbf{V}}_{l}(t, x)=\max _{y \in \mathbb{R}_{\geq 0}}\left\{\mathbf{v}_{0, l}(y)+t \mathbf{f}_{i}^{*}\left(\frac{x-y}{t}\right)\right\}$. In any case, the formulation of $\overline{\mathbf{V}}_{l}^{\text {in }}(t, x)$ and $\overline{\mathbf{V}}_{l}^{\text {out }}(t, x)$ includes information emanating from the initial datum. 


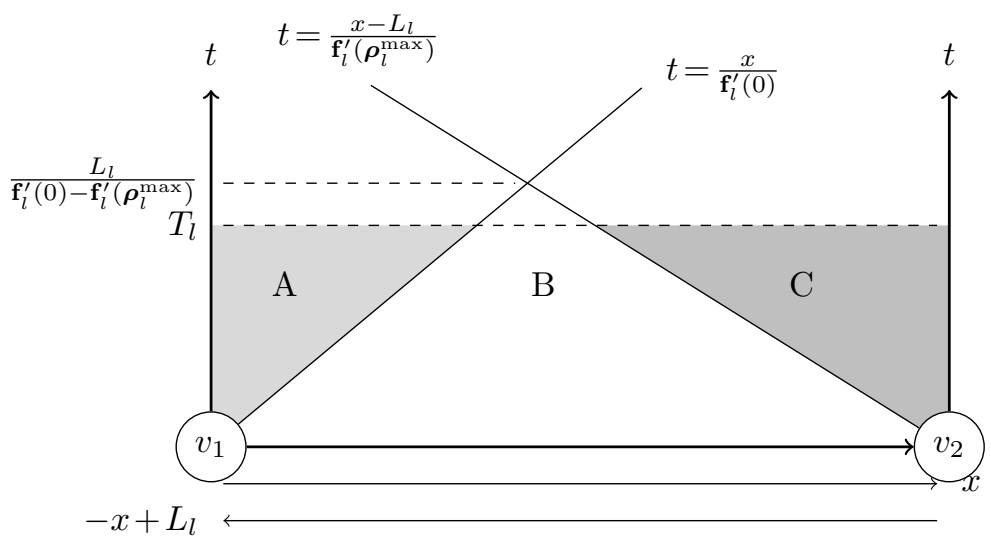

FIG. 7.1. Illustration of speed propagation argument on one link.

Since the solution $\overline{\mathbf{V}}_{l}(t, x)$ is Lipschitz-continuous, its spatial derivative can be be computed thanks to Rademacher's theorem (see for instance [34, Theorem 11.49]) or directly recalling the results in Theorems 4.1 and 4.2, thus obtaining the weak entropy solution to Eqs. (2.1) to (2.4).

\section{Further work and conclusion}

In this article we have rigorously built a framework providing weak solutions to the scalar conservation law on a network, obtaining regularity estimates on the HamiltonJacobi solutions. Nonetheless, from a traffic modeling point of view, this framework allows multiple origins but only a single destination. Adding multiple destinations for vehicles requires to add dynamics, keeping track of the different flows w.r.t. the different destinations, so called multi-commodity models. In addition to this extension, simulating the problem numerically seems appropriate to visualize the impact of the dynamics of buffers and routing functions on the solution. The developed HamiltonJacobi framework can thereby be used to implement a numerical scheme based on the considered fixed-point problem. Finally, the work detailed in Section 6 opens the door for Dynamic Traffic Assignment, i.e. optimizing the routing function with respect to the solution in real time or based on past information. This also continues the work in [33].

Acknowledgements. We gratefully acknowledge travel funding provided by the Inria Associated Team ORESTE (Optimal Reroute Strategies For Traffic Management) for cooperation between ACUMES project-team at Inria and Connected Corridors at UC Berkeley.

\section{REFERENCES}

[1] Y. Achdou, F. Camilli, A. Cutrì, and N. Tchou. Hamilton-Jacobi equations constrained on networks. NoDEA Nonlinear Differential Equations Appl., 20(3):413-445, 2013. 1

[2] Adimurthi, S. S. Ghoshal, R. Dutta, and V. G. D. Gowda. Existence and nonexistence of tv bounds for scalar conservation laws with discontinuous flux. Communications on Pure and Applied Mathematics, 64(1):84-115, 2011. 4.3

[3] Adimurthi, S. Mishra, and V. G. D. Gowda. Explicit Hopf-Lax type formulas for Hamilton-Jacobi equations and conservation laws with discontinuous coefficients. J. Differential Equations, 241(1):1-31, 2007. 1, 2.3.1 
[4] L. Ambrosio, N. Fusco, and D. Pallara. Functions of bounded variation and free discontinuity problems. Oxford Mathematical Monographs. The Clarendon Press, Oxford University Press, New York, 2000. 6

[5] D. Armbruster, P. Degond, and C. Ringhofer. A model for the dynamics of large queuing networks and supply chains. SIAM J. Appl. Math., 66(3):896-920, 2006. 1

[6] J.-P. Aubin, A. M. Bayen, and P. Saint-Pierre. Dirichlet problems for some Hamilton-Jacobi equations with inequality constraints. SIAM J. Control Optim., 47(5):2348-2380, 2008. 1

[7] C. Bardos, A. Y. le Roux, and J.-C. Nédélec. First order quasilinear equations with boundary conditions. Comm. Partial Differential Equations, 4(9):1017-1034, 1979. 3, 4.1, 4.2

[8] S. Boyd and L. Vandenberghe. Convex optimization. Cambridge University Press, Cambridge, 2004. 4.1

[9] A. Bressan and K. T. Nguyen. Conservation law models for traffic flow on a network of roads. Netw. Heterog. Media, 10(2):255-293, 2015. 1, 4.1, 4.1

[10] A. Bressan and K. T. Nguyen. Optima and equilibria for traffic flow on networks with backward propagating queues. Netw. Heterog. Media, 10(4):717-748, 2015. 1, 4.1

[11] A. Bressan and A. Nordli. The Riemann solver for traffic flow at an intersection with buffer of vanishing size. Netw. Heterog. Media, 12(2):173-189, 2017. 1, 4.1

[12] A. Bressan and F. Yu. Continuous Riemann solvers for traffic flow at a junction. Discrete Contin. Dyn. Syst., 35(9):4149-4171, 2015. 1

[13] E. S. Canepa and C. G. Claudel. Exact solutions to traffic density estimation problems involving the lighthill-whitham-richards traffic flow model using mixed integer programming. In 2012 15th International IEEE Conference on Intelligent Transportation Systems, pages 832-839. IEEE, 2012. 2.4.1

[14] C. G. Claudel and A. M. Bayen. Lax-Hopf based incorporation of internal boundary conditions into Hamilton-Jacobi equation. Part I: Theory. IEEE Trans. Automat. Control, 55(5):11421157, 2010. 1

[15] C. G. Claudel and A. M. Bayen. Lax-Hopf based incorporation of internal boundary conditions into Hamilton-Jacobi equation. Part II: Computational methods. IEEE Trans. Automat. Control, 55(5):1158-1174, 2010. 1

[16] R. M. Colombo, P. Goatin, and M. D. Rosini. On the modelling and management of traffic. ESAIM Math. Model. Numer. Anal., 45(5):853-872, 2011. 3

[17] M. G. Crandall and P.-L. Lions. Viscosity solutions of Hamilton-Jacobi equations. Trans. Amer. Math. Soc., 277(1):1-42, 1983. 1

[18] E. Cristiani and F. S. Priuli. A destination-preserving model for simulating Wardrop equilibria in traffic flow on networks. Netw. Heterog. Media, 10(4):857-876, 2015. 1

[19] L. C. Evans. Partial differential equations, volume 19 of Graduate Studies in Mathematics. American Mathematical Society, Providence, RI, second edition, 2010. 4.1, 4.3

[20] H. Frankowska. On LeFloch's solutions to the initial-boundary value problem for scalar conservation laws. J. Hyperbolic Differ. Equ., 7(3):503-543, 2010. 1

[21] M. Garavello and P. Goatin. The Cauchy problem at a node with buffer. Discrete Contin. Dyn. Syst., 32(6):1915-1938, 2012. 1, 2.3.2

[22] M. Garavello, K. Han, and B. Piccoli. Models for vehicular traffic on networks, volume 9 of AIMS Series on Applied Mathematics. American Institute of Mathematical Sciences (AIMS), Springfield, MO, 2016. 1

[23] M. Garavello and B. Piccoli. Source-destination flow on a road network. Commun. Math. Sci., 3(3):261-283, 2005. 1

[24] M. Garavello and B. Piccoli. Traffic flow on networks, volume 1 of AIMS Series on Applied Mathematics. American Institute of Mathematical Sciences (AIMS), Springfield, MO, 2006. Conservation laws models. 1

[25] M. Garavello and B. Piccoli. A multibuffer model for LWR road networks. In Advances in dynamic network modeling in complex transportation systems, volume 2 of Complex Netw. Dyn. Syst., pages 143-161. Springer, New York, 2013. 1

[26] S. Göttlich, M. Herty, and A. Klar. Network models for supply chains. Commun. Math. Sci., $3(4): 545-559,2005.1$

[27] M. Gugat, A. Keimer, G. Leugering, and Z. Wang. Analysis of a system of nonlocal conservation laws for multi-commodity flow on networks. Netw. Heterog. Media, 10(4):749-785, 2015. 1

[28] K. Han, B. Piccoli, and W. Szeto. Continuous-time link-based kinematic wave model: formulation, solution existence, and well-posedness. Transportmetrica B: Transport Dynamics, 4(3):187222, 2016. 2.4.1

[29] M. Herty, A. Klar, and B. Piccoli. Existence of solutions for supply chain models based on partial differential equations. SIAM J. Math. Anal., 39(1):160-173, 2007. 1

[30] M. Herty, J.-P. Lebacque, and S. Moutari. A novel model for intersections of vehicular traffic 
flow. Netw. Heterog. Media, 4(4):813-826, 2009. 1

[31] C. Imbert, R. Monneau, and H. Zidani. A Hamilton-Jacobi approach to junction problems and application to traffic flows. ESAIM Control Optim. Calc. Var., 19(1):129-166, 2013. 1

[32] K. T. Joseph and G. D. V. Gowda. Explicit formula for the solution of convex conservation laws with boundary condition. Duke Math. J., 62(2):401-416, 03 1991. 1, 4.1, 4.2, 4.3

[33] A. Keimer, N. Laurent-Brouty, F. Farokhi, H. Signargout, V. Cvetkovic, A. M. Bayen, and K. H. Johansson. Information patterns in the modeling and design of mobility management services. Proceedings of the IEEE, 106(4):554-576, 2018. 8

[34] G. Leoni. A first course in Sobolev spaces, volume 105 of Graduate Studies in Mathematics. American Mathematical Society, Providence, RI, 2009. 6, 7

[35] M. J. Lighthill and G. B. Whitham. On kinematic waves. II. A theory of traffic flow on long crowded roads. Proc. Roy. Soc. London. Ser. A., 229:317-345, 1955. 1

[36] B. G. Pachpatte. Inequalities for differential and integral equations, volume 197 of Mathematics in Science and Engineering. Academic Press, Inc., San Diego, CA, 1998. 5

[37] P. I. Richards. Shock waves on the highway. Operations Res., 4:42-51, 1956. 1

[38] S. Samaranayake, W. Krichene, J. Reilly, M. L. D. Monache, P. Goatin, and A. Bayen. Discretetime system optimal dynamic traffic assignment (SO-DTA) with partial control for physical queuing networks. Transportation Science, 52(4):982-1001, 2018. 1

[39] E. Zeidler. Applied Functional Analysis: Applications to Mathematical Physics. Applied Mathematical Sciences. Springer New York, 1995. 4.4 